\title{
CRESCITA DELLA PRODUTTIVITÀ, PROGRESSO TECNICO E IMPIEGO DEL LAVORO NELLE IMPRESE MANIFATTURIERE ITALIANE: 1996-2006
}

\section{PRODUCTIVITY GROWTH, TECHNICAL PROGRESS AND LABOUR USE IN ITALIAN MANUFACTURING FIRMS: 1996-2006}

\author{
Enrico Tundis ${ }^{*}$,Enrico Zaninotto ${ }^{* *}$, Roberto Gabriele ${ }^{* * *}$, Sandro Trento ${ }^{* * * *}$
}

\begin{abstract}
The article analyses the productivity dynamics of a sample of Italian manufacturing firms in the period 1996-2006. The first part computes Malmquist measures of productivity using non parametric techniques. Our measures show that the low rate of growth in productivity is due to the different models used to adapt to the changes in the competitive environment. Up to 2000, all the firms attempted to improve efficiency, whereas technical progress is absent. After 2000, there is evidence that a group of firms advance the technical frontier, while many firms lag behind and increase the frontier gap. The second part of the article correlates the observed measures of productivity to some characteristics of the employed labor. The hypothesis that productive performances could be put in relation to the two tiers of the labor markets resulting from the reforms adopted at the end of the century is supported. Highly productive firms use a higher share of skilled labor and fewer part-time workers. The reverse is true for less efficient firms.
\end{abstract}

Keywords: total factor productivity, Italian manufacturing, Malmquist index, DEA, two tiers labour market

Parole chiave: produttività totale dei fattori, settore manifatturiero italiano, indice di Malmquist, DEA, mercato del lavoro stratificato

Jel classification: L16, L60, D24, C14

Received: 3.5 .2012

Final revision received: 8.8.2012

* Università degli studi di Trento, Dipartimento di economia e management; enrico.tundis@unitn.it.

${ }^{* *}$ Università degli studi di Trento, Dipartimento di economia e management; enrico.zaninotto@unitn.it.

*** Università degli studi di Trento, Dipartimento di economia e management; roberto.gabriele@unitn.it.

**** Università degli studi di Trento, Dipartimento di economia e management; sandro.trento@unitn.it. 


\section{Introduzione}

Da vari anni si è sviluppato un dibattito sulla dinamica della produttività in Italia, che in buona parte si è intrecciato con una serie di analisi sul rallentamento della crescita potenziale della nostra economia che hanno spesso sottolineato il pericolo di un "declino dell' economia italiana" (Ciocca, 2004; D'Antonio e Scarlato, 2004; Toniolo, 2004).

Secondo alcuni le differenze nella crescita fra l'Italia e altri paesi avanzati sarebbero da ricondurre ai diversi livelli di produttività multifattoriale, dovuti a propria volta alla minore capacità d'innovazione e di progresso tecnico. Il gap tra Italia e altri paesi avanzati sarebbe dovuto sia alla minore produzione di nuove tecnologie sia al loro più basso utilizzo (Daveri e Jona-Lasino, 2005; Faini, 2004). Questa minore capacità di sfruttamento del paradigma tecnologico fondato sulle tecnologie digitali sarebbe a sua volta legata a tratti strutturali del nostro sistema produttivo come la ridotta dimensione media delle imprese, la specializzazione in settori tradizionali, la minore qualità della manodopera.

La questione dimensionale e settoriale rimane uno dei temi cruciali nel dibattito in questione. Foresti, Guelpa e Trenti (2007) hanno nondimeno posto in luce come la specializzazione settoriale abbia avuto un ruolo molto limitato nello spiegare il gap di produttività tra Italia e altri paesi e l'andamento della struttura dimensionale avrebbe determinato solo in parte molto ridotta il peggioramento della produttività dell'Italia rispetto a Francia, Germania e Regno Unito. Secondo questi autori sarebbero stati fattori di contesto a influire negativamente sulla dinamica della produttività delle imprese italiane: inefficienza nei servizi alle imprese; pubblica amministrazione inadeguata, minore sforzo in ricerca e sviluppo.

Le istituzioni e le regole del mercato del lavoro sono state anch'esse prese in considerazione tra le spiegazioni possibili del modesto andamento della produttività in Italia. Le opportunità innovative e la possibilità di adottare e di impiegare al meglio le nuove tecnologie sarebbero legate, secondo alcuni studiosi, alle caratteristiche del mercato del lavoro e ai costi di aggiustamento del fattore lavoro (Blanchard e Landier, 2002; Tronti, 2010). Su una linea analoga, Boeri e Garibaldi (2007) sostengono che all'origine del declino della produttività (del lavoro) e dell'aumento della sua dispersione stia la riforma del lavoro e la costituzione di un modello stratificato dello stesso, con una componente rigida e una flessibile. Dopo il 2000 il prodotto sarebbe cresciuto in Italia quasi unicamente a seguito di un aumento delle ore lavorate totali, favorito dalla flessibilità maggiore introdotta nel nostro paese con le varie riforme del mercato del lavoro (Daveri, 2008). Altri studiosi tuttavia sembrano avere individuato un legame negativo tra maggiore flessibilità e produttività dovuta ai minori incentivi a investire in capitale umano specifico (Lucidi, 2008). Faini e 
Sapir (2005) hanno sostenuto che la minore capacità innovativa delle imprese italiane e la connessa incapacità di introdurre nuove tecnologie indispensabili per accrescere la produttività sarebbero dovute all'insufficiente offerta di lavoratori istruiti e a elevata qualificazione.

Se da un lato si sostiene che è soprattutto la caduta della produttività totale dei fattori la variabile che spiega la stagnazione dell'economia italiana (Brandolini e Cipollone, 2001; IMF, 2007), questa caduta sarebbe stata a sua volta controbilanciata da un maggiore impiego del fattore lavoro a seguito delle riforme effettuate. Ma vi è anche chi ha sostenuto che il rapporto tra capitale e lavoro sarebbe rimasto stabile e che quindi il rallentamento della produttività non potrebbe essere stato dovuto a un maggiore ricorso al fattore lavoro (OECD, 2007).

Di recente sono state avanzate diverse critiche alle stesse evidenze di caduta della produttività. In primo luogo, si è criticato il modo utilizzato in prevalenza per misurare la produttività del lavoro e totale dei fattori, basato su scomposizioni ottenute con la contabilità della crescita: esso infatti è soggetto ad assunzioni molto rigide sulla natura della funzione di produzione; in secondo luogo, si è rilevato che le procedure di deflazione impiegate possono avere sovrastimato il deflatore dei prodotti esportati accentuando in questo modo la presunta caduta delle esportazioni italiane (Brandolini e Bugamelli, 2009; Bugamelli, 2007). Infine, alla luce della crisi, si è fatto notare come i confronti con paesi come il Regno Unito e gli Stati Uniti possano essere stati viziati dall'effetto sul calcolo del prodotto interno della bolla immobiliare e finanziaria particolarmente grande in quei sistemi.

Gli studi aggregati giungono a giudizi piuttosto pessimistici sui tratti strutturali dell'economia italiana.

Nei contributi più recenti, l'attenzione si è spostata invece sull'eterogeneità dei comportamenti delle imprese. Sotto un certo profilo, l'evidenza di una forte eterogeneità di comportamenti porta a giudizi meno negativi sullo stato dell'economia italiana, in quanto mette in luce come siano presenti vaste aree del sistema produttivo capaci di innovare e di rispondere alle nuove condizioni competitive. Tuttavia le domande si spostano sulla ricerca delle cause di comportamenti molto difformi e sull'analisi degli effetti che un ampio e persistente divario potrebbe avere sulle performance di lungo periodo del sistema e sul suo posizionamento competitivo. Dosi et al. (2011) parlano, a questo proposito, di «turbolence underneath the big calm». Analizzando un campione di oltre centomila imprese di tutti i settori economici, trovano un aumento della dispersione della produttività del lavoro tra il 1989 e il 2004; la dispersione crescerebbe successivamente all'introduzione dell'euro, dando luogo a una sorta di neodualismo tra imprese dinamiche e imprese che non tengono il passo della crescita produttiva. A conclusioni simili pervengono Bugamelli, Schivardi e Zizza 
(2010), sostenendo che l'ingresso nell'euro non avrebbe agito tanto sulla riallocazione delle imprese tra settori, quanto nel determinare cambiamenti interni alle imprese e che tale effetto sia stato superiore nelle imprese che maggiormente si erano avvantaggiate in passato di svalutazioni competitive.

Questo lavoro si colloca all'interno di questa prospettiva tentando di conciliare la visione pessimistica emergente dalle analisi aggregate, con le evidenze di un'ampia eterogeneità di comportamenti da parte delle imprese. Al tempo stesso si supporta la tesi che il declino di produttività sia da mettere in relazione con le riforme del mercato del lavoro, in una direzione analoga a quella degli studi già citati di Boeri e Garibaldi (2007), Lucidi (2008) e Tronti (2010), ma rispetto a questi studi che sottolineano l'effetto aggregato delle "riforme al margine", nel nostro lavoro sosteniamo l'idea che il dualismo nel mercato del lavoro abbia alimentato una divergenza di comportamenti tra le imprese. L'effetto delle riforme non sarebbe pertanto uniforme; viceversa, la nostra indagine empirica supporta l'idea che, con l'ingresso nell'euro e le riforme introdotte nel mercato del lavoro, il nostro sistema industriale abbia assunto un carattere dualistico, con un gruppo di imprese che - dopo uno shock iniziale - riprende un modello di crescita basato sull'avanzamento tecnologico, e un gruppo che invece si adatta al nuovo contesto produttivo attraverso l'impiego di lavoro meno qualificato e stabile, sfruttando le fasce meno protette del mercato del lavoro.

L'analisi fa riferimento a una base di dati di impresa originale, costruita collegando i dati di bilancio AIDA con dati INPS (Istituto nazionale di previdenza sociale) relativi ai lavoratori impiegati dalle imprese. Essa si svolge in due parti. La prima si basa sulla valutazione della produttività totale dei fattori delle imprese attraverso misure di distanza rispetto alla frontiera produttiva settoriale e alla loro variazione nel tempo (indice di Malmquist). La scomposizione di questo indicatore mette in luce diversi aspetti della bassa dinamica della produttività delle imprese. Anzitutto colloca la fase maggiormente negativa della dinamica della produttività totale dei fattori negli ultimi anni del decennio 1990 quando, esauritasi la spinta delle svalutazioni competitive dei primi anni del decennio, l'industria italiana si trova a dover affrontare l'accresciuta concorrenza dei paesi emergenti in una condizione di cambi rigidi. In secondo luogo, mostra come negli anni successivi ci sia una debole ripresa della produttività che però è il risultato di una crescita vivace delle imprese poste sulla frontiera, e di un allontanamento dalla stessa di un secondo gruppo, la cui efficienza media diminuisce. Il fenomeno di spostamento della frontiera e riduzione dell'efficienza media, da noi rilevato a partire dal 2000 e soprattutto all'uscita della crisi del 2001, trova conferme in altri studi. Aiello, Pupo e Ricotta (2009), pur impiegando tecniche diverse, individuano una caduta della produttività tra il 1996 e il 2003, con una ripresa nei due anni successivi. Una serie di lavori, poi, conferma l'avvio di un profondo processo di ristrutturazio- 
ne produttiva nel sistema manifatturiero italiano in seguito all'introduzione dell'euro: va in quella direzione il già citato studio di Bugamelli, Schivardi e Zizza (2010) che sottolinea il ruolo dei cambiamenti interni al sistema produttivo rispetto alle componenti di riallocazione between e conferma l'aumento della dispersione della produttività del lavoro. Un aumento della dispersione delle performances delle imprese è rilevato anche da Dosi et al. (2011) che segnalano un ingrossamento della coda di sinistra della distribuzione della produttività del lavoro delle imprese seguito all'introduzione dell'euro, anche se (perlomeno in termini di produttività del lavoro) rilevano una crescita maggiore nel quinquennio precedente il 2000 rispetto a quello successivo.

La seconda parte dello studio usa gli indicatori di efficienza generati per il calcolo dell'indice di Malmquist nell'analisi delle caratteristiche delle imprese che presentano diversi sentieri di crescita della produttività. A questo scopo, gli andamenti rilevati nella prima parte dello studio, sono utilizzati per ottenere una classificazione delle imprese basata sulla loro dinamica di produttività. L'appartenenza a ogni singola categoria è stata messa in relazione con alcune caratteristiche delle imprese stesse, relative in particolare al costo e alla struttura del lavoro. Le imprese che appartengono al gruppo meno dinamico, vale a dire quelle caratterizzate da una minore dinamica di produttività sembrano essere accomunate da alcune caratteristiche del lavoro impiegato: hanno un costo del lavoro inferiore, un minore rapporto tra impiegati e operai e un maggiore uso di lavoratori a part time. Questo risultato va nella direzione di rafforzare la linea interpretativa proposta da Boeri e Garibaldi (2007) che riconduce l'aumento della varianza della produttività al dualismo indotto nel mercato del lavoro dalle riforme introdotte a cavallo del secolo.

Nelle prossime sezioni procederemo alla descrizione del database impiegato (sezione uno); presenteremo quindi la metodologia di stima (sezione due) e i principali risultati dell'analisi della produttività e delle sue componenti (sezione tre); infine, nella sezione quattro classificheremo le imprese a seconda della loro dinamica di produttività e presenteremo i risultati della stima di un modello logit multinomiale per lo studio dei fattori associati all'appartenenza alle diverse categorie.

\section{La base di dati e le variabili impiegate}

Il database utilizzato nel presente lavoro è composto da informazioni relative ad un sottoinsieme delle società di capitale che operavano in Italia nel periodo 1996-2006 (SdC19962006). La fonte primaria delle informazioni raccolte è l'archivio AIDA prodotto da Bureau Van Dijk. Tale archivio contiene informazioni sulle voci di bilancio e su alcune caratteristiche qualitative e 
quantitative delle imprese, quali la data di costituzione, il numero di addetti e la localizzazione geografica.

Un problema importante dei dati AIDA concerne la qualità e l'alto numero di dati mancanti sull'occupazione nelle singole imprese. Il secondo passo nella costruzione del nostro database è consistito, dunque, nell'integrazione dei dati di bilancio con informazioni maggiormente affidabili sul numero medio annuo degli addetti relativo a tutte le imprese del nostro campione. Tali informazioni provengono dall'archivio sulle forze lavoro dell'INPS. Dal dataset così costruito, sono state eliminate infine le imprese multi-impianto.

Si è così ottenuto un panel bilanciato di settemilasettecentododici piccole e medie società di capitali mono-impianto (ottantaquattromila e ottocentotrentadue osservazioni). La tab. 1 confronta i dati sulla distribuzione delle società di capitale e relativi addetti per sottosezione ATECO-2002 del nostro dataset con i corrispondenti dati Istat relativi all' $8^{\circ}$ Censimento industria e servizi del 2001. Come si può notare, la distribuzione settoriale del nostro dataset rispecchia la distribuzione delle società di capitale fotografate dall'Istat nel 2001, anno centrale dell'intervallo di tempo analizzato. Sono rappresentati con un maggiore peso, in termini di imprese, i settori della gomma, del metallo e delle macchine e apparecchiature meccaniche, i primi anche in termini di addetti. Sottorappresentati sono la carta, gli apparecchi elettrici, sia in termini di imprese che di addetti. Il tessile è sovarappresentato in termini di addetti.

Tab. 1 - Distribuzione delle imprese e degli addetti per settore di attività (2001)

\begin{tabular}{|c|c|c|c|c|c|c|c|c|}
\hline & \multicolumn{4}{|c|}{ Imprese } & \multicolumn{4}{|c|}{ Addetti } \\
\hline & \multicolumn{2}{|c|}{ Istat ${ }^{a}$} & \multicolumn{2}{|c|}{ Ns. database ${ }^{b}$} & \multicolumn{2}{|c|}{ Istat $^{a}$} & \multicolumn{2}{|c|}{ Ns. database $e^{b}$} \\
\hline & Numero & $\%$ & Numero & $\%$ & Numero & $\%$ & Numero & $\%$ \\
\hline Alimentare e tabacco & 8.328 & 7,2 & 564 & 7,3 & 220.922 & 6,8 & 25.404 & 6,2 \\
\hline Tessile e abbigliamento & 13.929 & 12,0 & 911 & 11,8 & 352.291 & 10,8 & 51.645 & 12,6 \\
\hline Cuoio & 4.869 & 4,2 & 365 & 4,7 & 113.573 & 3,5 & 19.971 & 4,8 \\
\hline Legno & 3.281 & 2,8 & 204 & 2,6 & 56.284 & 1,7 & 9.071 & 2,2 \\
\hline Carta, stampa e editoria & 9.838 & 8,5 & 479 & 6,2 & 178.708 & 5,5 & 21.419 & 5,2 \\
\hline Raffinerie di petrolio & 352 & 0,3 & 22 & 0,3 & 24.192 & 0,7 & 1.045 & 0,2 \\
\hline Chimica & 3.797 & 3,3 & 309 & 4,0 & 197.340 & 6,0 & 17.313 & 4,2 \\
\hline Gomma e plastica & 5.993 & 5,2 & 492 & 6,3 & 175.330 & 5,4 & 26.858 & 6,5 \\
\hline Minerali non metalliferi & 6.399 & 5,5 & 433 & 5,6 & 175.035 & 5,4 & 21.676 & 5,3 \\
\hline Metallo e prodotti in metallo & 20.545 & 17,7 & 1445 & 18,7 & 503.712 & 15,4 & 77.814 & 19,0 \\
\hline Macchine e app. meccanici & 15.879 & 13,7 & 1137 & 14,7 & 498.507 & 15,3 & 62.991 & 15,3 \\
\hline App. elettrici e di precisione & 11.291 & 9,7 & 574 & 7,4 & 344.198 & 10,5 & 31.104 & 7,6 \\
\hline Mezzi di trasporto & 2.697 & 2,3 & 161 & 2,1 & 253.778 & 7,8 & 10.691 & 2,6 \\
\hline Altre industrie manifatturiere & 8.716 & 7,5 & 616 & 7,9 & 174.104 & 5,3 & 32.288 & 7,8 \\
\hline Manifattura & 115.914 & 100,0 & 7.712 & 100,0 & 3.267 .974 & 100,0 & 409.290 & 100,0 \\
\hline
\end{tabular}

${ }^{a}$ valori riferiti all'intera popolazione delle società di capitale: multi e mono-impianto.

${ }^{\mathrm{b}}$ valori riferiti all'intero campione.

Fonte: nostre elaborazioni su dati SdC19962006; Istat (2001). 
E. Tundis, E. Zaninotto, R. Gabriele, S. Trento Productivity of Italian firms: 1996-2006

Tab. 2 - Variabili di input e output utilizzate per la stima della produttività. Anno 2006

\begin{tabular}{|c|c|c|c|c|c|c|}
\hline Settore & & $\begin{array}{l}\text { Fatturato } \\
(\text { mgl. } €)\end{array}$ & $\begin{array}{l}\text { Addetti } \\
\text { (unità) }\end{array}$ & $\begin{array}{c}\text { Servizi } \\
(m g l . €)\end{array}$ & $\begin{array}{l}\text { Materie prime } \\
(\mathrm{mgl} €)\end{array}$ & $\begin{array}{l}\text { Imm. mat. } \\
\text { (mgl. } €)\end{array}$ \\
\hline Alimentare e tabacco & Media & $15.669,5$ & 44,31 & $2.652,3$ & $10.263,4$ & $3.490,4$ \\
\hline \multirow[t]{2}{*}{ Tessile e abbigliamento } & $\begin{array}{l}\text { Dev. std. } \\
\text { Media }\end{array}$ & $\begin{array}{r}25.117,2 \\
9.109,3\end{array}$ & $\begin{array}{l}55,56 \\
50,33\end{array}$ & $\begin{array}{l}4.747,6 \\
2.941,1\end{array}$ & $\begin{array}{r}18.072,1 \\
3.931,2\end{array}$ & $\begin{array}{l}5.209,3 \\
1.629,7\end{array}$ \\
\hline & Dev. std. & $13.017,2$ & 65,79 & $4.488,2$ & $6.346,0$ & $3.459,8$ \\
\hline \multirow[t]{2}{*}{ Cuoio } & Media & $10.510,2$ & 45,70 & $2.647,6$ & $5.899,3$ & $1.186,6$ \\
\hline & Dev. std. & $13.650,8$ & 48,80 & $3.554,8$ & $8.682,2$ & $1.738,5$ \\
\hline \multirow[t]{2}{*}{ Legno } & Media & $8.185,0$ & 43,38 & $1.749,4$ & $4.425,8$ & $2.030,9$ \\
\hline & Dev. std. & $9.979,3$ & 40,97 & $2.824,6$ & $5.586,4$ & $3.055,5$ \\
\hline \multirow[t]{2}{*}{ Carta, stampa e editoria } & Media & $8.662,5$ & 43,94 & $2.155,0$ & $3.979,7$ & $2.153,4$ \\
\hline & Dev. std. & $9.640,1$ & 40,79 & $2.611,1$ & $5.698,8$ & $3.512,9$ \\
\hline \multirow[t]{2}{*}{ Raffinerie di petrolio } & Media & $26.414,5$ & 51,24 & $2.561,3$ & $18.993,9$ & $8.176,1$ \\
\hline & Dev. std. & $42.909,3$ & 70,51 & $3.856,4$ & $37.197,5$ & $25.167,8$ \\
\hline \multirow[t]{2}{*}{ Chimica } & Media & $18.540,3$ & 59,50 & $4.234,7$ & $9.706,4$ & $2.764,4$ \\
\hline & Dev. std. & $38.739,3$ & 75,74 & $12.159,0$ & $18.641,5$ & $4.774,9$ \\
\hline \multirow[t]{2}{*}{ Gomma e plastica } & Media & $10.846,8$ & 54,77 & $2.055,2$ & $5.898,8$ & $2.484,2$ \\
\hline & Dev. std. & $16.665,0$ & 71,06 & $2.981,7$ & $10.270,9$ & $7.319,7$ \\
\hline \multirow[t]{2}{*}{ Minerali non metalliferi } & Media & $10.014,8$ & 49,65 & $2.429,3$ & $4.704,8$ & $2.459,3$ \\
\hline & Dev. std. & $14.122,2$ & 59,21 & $4.190,4$ & $6.737,6$ & $3.500,9$ \\
\hline \multirow[t]{2}{*}{ Metallo e prodotti in metallo } & Media & $13.774,9$ & 54,73 & $2.292,0$ & $8.215,4$ & $2.330,1$ \\
\hline & Dev. std. & $55.741,7$ & 63,06 & $4.576,7$ & $50.413,3$ & $4.813,5$ \\
\hline \multirow[t]{2}{*}{ Macchine e app. meccanici } & Media & $11.097,2$ & 56,81 & $2.245,3$ & $5.601,1$ & $1.556,4$ \\
\hline & Dev. std. & $16.495,7$ & 66,52 & $3.246,3$ & $9.926,1$ & $2.514,6$ \\
\hline \multirow[t]{2}{*}{ App. elettrici e di precisione } & Media & $9.987,7$ & 52,98 & $1.936,2$ & $5.138,7$ & $1.258,7$ \\
\hline & Dev. std. & $16.243,5$ & 52,34 & $2.102,5$ & $12.652,7$ & $2.210,3$ \\
\hline \multirow[t]{2}{*}{ Mezzi di trasporto } & Media & $14.649,7$ & 63,88 & $2.836,3$ & $8.344,9$ & $2.115,5$ \\
\hline & Dev. std. & $28.463,5$ & 70,80 & $4.474,4$ & $21.173,8$ & $2.719,7$ \\
\hline \multirow[t]{2}{*}{ Altre industrie manifatturiere } & Media & $8.868,7$ & 49,52 & $2.141,6$ & $4.647,5$ & $1.752,0$ \\
\hline & Dev. std. & $11.117,8$ & 49,03 & $3.332,0$ & $6.582,7$ & $2.960,9$ \\
\hline
\end{tabular}

Fonte: nostre elaborazioni su dati SdC19962006.

La costruzione delle variabili di input e di output per il calcolo delle variazioni intertemporali della produttività è basata su dati di bilancio, con l'eccezione del dato sull'impiego del lavoro. I dati grezzi sono stati successivamente elaborati, corretti e deflazionati al fine di ottenere valori reali. In questo studio si sono utilizzati deflatori settoriali costruiti ad hoc utilizzando dati Istat.

Le variabili di input e di output costruite dei dati primari di bilancio per l'analisi della produttività sono:

1. Fatturato (output): valore dei ricavi delle vendite e delle prestazioni al netto della variazione delle rimanenze di prodotti in corso di lavorazione, semilavorati e finiti e della variazione dei lavori in corso su ordinazione. Il deflatore per la variabile Fatturato è stato costruito elaborando le serie storiche nazionali della produzione.

2. Costo per le materie prime (input): costi della produzione per materie prime, sussidiarie, di consumo e servizi al netto della variazione delle rima- 
nenze di materie prime, sussidiarie, di consumo e merci. Il deflatore settoriale per questa variabile è stato costruito utilizzando i deflatori della produzione ponderati per un sistema di pesi calcolati come media dei coefficienti di colonna delle matrici input/output relative all'anno 2001 per un set di regioni italiane.

3. Costo per i servizi (input): costo della produzione per servizi deflazionato mediante il deflatore settoriale della produzione.

4. Stima dello stock di capitale (input): per costruire questa variabile si è utilizzato come punto di riferimento il valore storico delle immobilizzazioni materiali e si è adottato un metodo derivato dall'inventario permanente (vedi Pedrotti, Tundis e Zaninotto, 2008).

5. Lavoro (input): il lavoro impiegato è misurato come numero medio annuo degli addetti relativo a ogni impresa. La fonte del dato occupazionale è l'INPS. In tab. 2 sono riportate alcune statistiche descrittive sulle variabili di input e output relative al 2006, anno finale di osservazione nel nostro dataset.

\section{La misura della produttività e la sua scomposizione}

Per l'analisi si è proceduto alla misurazione della variazione della produttività totale dei fattori mediante indici di Malmquist e alla loro scomposizione in un contesto di stima non parametrico. L'analisi è basata su stime non parametriche in considerazione della possibilità che queste offrono di non condizionare l'analisi a una precisa specificazione della funzione di produzione che, soprattutto in un contesto di confronto intersettoriale, porrebbe vincoli troppo rigidi. Tra i metodi di stima non parametrici, poi, si è impiegata la data envelopment analysis (DEA). ${ }^{1} \mathrm{Si}$ è cercato peraltro di porre rimedio ai limiti più evidenti delle analisi non parametriche, ricorrendo a procedure di stima DEA robuste rispetto alla presenza di outliers.

Nel caso più semplice di imprese che utilizzano un input $x$ per produrre un solo output $y$, la variazione della produttività totale dei fattori della generica impresa $i$ sarà semplicemente definita come il rapporto tra la produttività totale, calcolata come rapporto del singolo output sul singolo input, in due istanti $t$ e $t+k$ :

$\Delta \operatorname{TFP}_{i}=\frac{\left(y_{i}^{t+k} / x_{i}^{t+k}\right)}{\left(y_{i}^{t} / x_{i}^{t}\right)}$

Nella condizione più generale in cui le imprese utilizzano più input per produrre più output, la variazione della produttività totale dei fattori di un'im-

1. Tale metodologia riscuote sempre più interesse grazie anche ai recenti lavori che ne hanno dimostrato le proprietà statistiche (Simar e Wilson, 2008). 
presa $i$ può essere misurata mediante un indice di Malmquist ottenuto come rapporto della distanza dell'impresa dalla frontiera produttiva in due istanti di tempo diversi.

Una funzione di distanza è definita in relazione o agli output o agli input. L'approccio utilizzato nel nostro lavoro è quello output-oriented, quindi si considerano le frontiere delle possibilità produttive dati gli input. ${ }^{2}$ Formalmente, si consideri il caso generale di un'impresa $i$ che al tempo $t$ produce un vettore di output $\mathbf{y}_{i}^{t} \in \mathfrak{R}_{+}^{M}$, dato un vettore di input $\mathbf{x}_{i}^{t} \in \mathfrak{R}_{+}^{S}$. Una funzione di distanza può dunque essere definita, data una tecnologia produttiva $T^{t}=\left\{\left(\mathbf{y}^{t}, \mathbf{x}^{t}\right): \mathbf{x}^{t}\right.$ può produrre $\left.\mathbf{y}^{t}\right\}$, come segue (vedi Coelli et al., 2005):

$D_{o}^{t}\left(\mathbf{y}_{i}^{t}, \mathbf{x}_{i}^{t}\right)=\inf _{\theta_{i}}\left\{\theta_{i}:\left(\mathbf{x}_{i}^{t}, \frac{\mathbf{y}_{i}^{t}}{\theta_{i}}\right) \in T^{t}\right\}$

Lo scalare $\theta_{i} \in(0,1]$ nella formula [2] individua la distanza (o efficienza) in termini di massimo incremento ammissibile dell'output $\mathbf{y}_{i}^{t}$ dato l'input $\mathbf{x}_{i}^{t}$, cioè tale che la combinazione produttiva $\left(\mathbf{x}_{i}^{t}, \mathbf{y}_{i}^{t} / \theta_{i}\right)$ sia sulla frontiera dell'insieme delle combinazioni produttive ammissibili al tempo $t$. Pertanto un'impresa apparterrà alla frontiera se e solo se $D_{o}^{t}\left(\mathbf{y}_{i}^{t}, \mathbf{x}_{i}^{t}\right)=1$.

È possibile definire la relazione tra la variazione della produttività totale dei fattori di un'impresa e la variazione nel tempo delle misure di distanza della combinazione di input e output che la rappresenta. Färe et al. (1994) definirono un indice di Malmquist considerando una media geometrica delle distanze riferite alla frontiera al tempo $t$ e $t+k$ sotto l'assunzione di rendimenti costanti di scala (CRS). L'indice di Malmquist orientato agli output definito da Färe et al. (1994) è formalizzato come segue: ${ }^{3}$

$M_{o}\left(\mathbf{x}^{t+k}, \mathbf{y}^{t+k}, \mathbf{x}^{t}, \mathbf{y}^{t}\right)=\left[\frac{D_{o}^{t}\left(\mathbf{x}^{t+k}, \mathbf{y}^{t+k} \mid C R S\right)}{D_{o}^{t}\left(\mathbf{x}^{t}, \mathbf{y}^{t} \mid C R S\right)} \times \frac{D_{o}^{t+k}\left(\mathbf{x}^{t+k}, \mathbf{y}^{t+k} \mid C R S\right)}{D_{o}^{t+k}\left(\mathbf{x}^{t}, \mathbf{y}^{t} \mid C R S\right)}\right]^{1 / 2}$

Se l'indice assume valori minori di uno significa che dal periodo $t$ al periodo $t+k$ si è verificato, per quella particolare impresa, un calo di produttività. Se, invece, l'indice assume valori superiori a uno significa che si è verificato un incremento di produttività. Se l'indice è uguale a uno vuol dire che la produttività nei due periodi è rimasta invariata.

L'indice di Malmquist si presta a una serie di scomposizioni che permettono di evidenziare il ruolo di diverse fonti della crescita della produttività. Una prima scomposizione possibile mette in luce le componenti di efficienza e

2. In alternativa è possibile utilizzare un approccio input-oriented. Nell'ipotesi di rendimenti di scala costanti, le misure di distanza calcolate con i due approcci si equivalgono.

3. L'indice di Malmquist, così come le sue componenti che verranno presentate in seguito, si riferisce ovviamente alla singola impresa. Tuttavia, l'indicatore $i$ è omesso per semplificare la notazione. 
cambiamento tecnologico della variazione della produttività. Entrambe le componenti possono essere poi ulteriormente scomposte isolando gli effetti della scala produttiva. Infine, una terza scomposizione evidenzia il ruolo della composizione dei fattori produttivi nel cambiamento tecnologico. Presentiamo di seguito le tre scomposizioni impiegate nel nostro lavoro.

Quanto alla prima scomposizione, mediante semplici trasformazioni algebriche l'indice di Malmquist assume la seguente forma (Färe et al., 1994):

$M_{o}\left(\mathbf{x}^{t+k}, \mathbf{y}^{t+k}, \mathbf{x}^{t}, \mathbf{y}^{t}\right)=\frac{D_{o}^{t+k}\left(\mathbf{x}^{t+k}, \mathbf{y}^{t+k} \mid C R S\right)}{D_{o}^{t}\left(\mathbf{x}^{t}, \mathbf{y}^{t} \mid C R S\right)} \times\left[\frac{D_{o}^{t}\left(\mathbf{x}^{t+k}, \mathbf{y}^{t+k} \mid C R S\right)}{D_{o}^{t+k}\left(\mathbf{x}^{t+k}, \mathbf{y}^{t+k} \mid C R S\right)} \times \frac{D_{o}^{t}\left(\mathbf{x}^{t}, \mathbf{y}^{t} \mid C R S\right)}{D_{o}^{t+k}\left(\mathbf{x}^{t}, \mathbf{y}^{t} \mid C R S\right)}\right]^{1 / 2}=$
$=E F F C H_{t, t+k} \times T E C H_{t, t+k}$

La prima componente $\left(E F F C H_{t, t+k}\right)$ della parte destra dell'equazione è uguale al reciproco del rapporto delle misure dell'efficienza tecnica "alla Farrell" e cattura l'effetto della variazione di efficienza da $t$ a $t+k$. La seconda componente $\left(\mathrm{TECH}_{t, t+k}\right)$ cattura invece il cambiamento tecnologico cioè il mutamento tecnologico dovuto al migliore impiego dei fattori, calcolato come media geometrica dello spostamento della frontiera produttiva per i livelli di input $x^{t}$ e $x^{t+k}$. Per entrambe le componenti isolate, valori minori di uno indicano un contributo negativo alla variazione di produttività tra i periodi $t$ e $t+k$; un valore superiore a uno indica un contributo positivo; infine il contributo è nullo se il valore assunto è uno. ${ }^{4}$

La seconda scomposizione proposta è relativa rispettivamente alla componente di efficienza e a quella del cambiamento tecnologico dell'indice di Malmquist. Riguardo alla componente di efficienza $\left(E F F C H_{t, t+k}\right)$, gli effetti di scala possono essere rilevati stimando la frontiera di produzione nell'ipotesi di rendimenti di scala variabili (VRS) e separando la variazione dell'efficienza rispetto alla frontiera calcolata nell'ipotesi di VRS (pura efficienza) dalla componente legata allo spostamento del punto di riferimento lungo la frontiera VRS rispetto a quella CRS (efficienza di scala). Nella scomposizione che ne risulta (Färe et al., 1994), si ha:

$$
\begin{aligned}
& M_{o}\left(\mathbf{x}^{t+k}, \mathbf{y}^{t+k}, \mathbf{x}^{t}, \mathbf{y}^{t}\right)=\left(\frac{D_{o}^{t+k}\left(\mathbf{x}^{t+k}, \mathbf{y}^{t+k} \mid V R S\right)}{D_{o}^{t}\left(\mathbf{x}^{t}, \mathbf{y}^{t} \mid V R S\right)}\right)^{t} \times\left(\frac{D_{o}^{t+k}\left(\mathbf{x}^{t+k}, \mathbf{y}^{t+k} \mid C R S\right)}{D_{o}^{t+k}\left(\mathbf{x}^{t+k}, \mathbf{y}^{t+k} \mid V R S\right)} \times \frac{D_{o}^{t}\left(\mathbf{x}^{t}, \mathbf{y}^{t} \mid V R S\right)}{D_{o}^{t}\left(\mathbf{x}^{t}, \mathbf{y}^{t} \mid C R S\right)}\right) \times \\
& \times\left[\frac{D_{o}^{t}\left(\mathbf{x}^{t+k}, \mathbf{y}^{t+k} \mid C R S\right)}{D_{o}^{t+k}\left(\mathbf{x}^{t+k}, \mathbf{y}^{t+k} \mid C R S\right)} \times \frac{D_{o}^{t}\left(\mathbf{x}^{t}, \mathbf{y}^{t} \mid C R S\right)}{D_{o}^{t+k}\left(\mathbf{x}^{t}, \mathbf{y}^{t} \mid C R S\right)}\right]^{1 / 2}=P E F F C H_{t, t+k} \times S C H_{t, t+k} \times T E C H_{t, t+k}
\end{aligned}
$$

Il primo termine a destra è la componente di pura efficienza della crescita di produttività $\left(\mathrm{PEFFCH}_{t, t+k}\right)$ calcolata rispetto alla frontiera a rendimenti va-

4. È utile notare che l'utilizzo di frontiere intertemporali, costruite con le osservazioni disponibili rispettivamente agli istanti di tempo $t$ e $t+k$, fa si che in alcuni casi i valori delle distanze $D_{o}^{p}\left(\mathbf{x}^{q}, \mathbf{y}^{q}\right)$ possano essere maggiori di uno. In particolare, ciò può accadere quando il progresso tecnico rende impossibile produrre $\mathbf{y}^{t+k}$ con gli input $\mathbf{x}^{t+k}$ data la tecnologia del periodo $t$. 
riabili; il secondo termine $\left(\mathrm{SCH}_{t, t+k}\right)$ esprime i guadagni o le perdite di produttività dovute al fatto che il tratto di riferimento della frontiera rilevante per una data impresa si sposta verso punti della frontiera stessa più o meno vicini al punto di tangenza tra la frontiera a rendimenti costanti e quella a rendimenti variabili (most productive scale size) e rappresenta dunque i guadagni (valori maggiori di uno) o le perdite (valori minori di uno) dovute alla scala produttiva; il terzo è la componente tecnologica dell'indice di Malmquist isolata in precedenza, calcolata nell'ipotesi di rendimenti constanti $\left(\mathrm{TECH}_{t, t+k}\right)$.

La componente tecnologica può anch'essa essere ulteriormente scomposta. $\mathrm{TECH}_{t, t+k}$ rappresenta, come si è detto, lo spostamento della frontiera a rendimenti costanti. Ma tale spostamento è a propria volta dipendente dallo spostamento della frontiera a rendimenti variabili $\left(\mathrm{PTECH}_{t, t+k}\right)$, e dal cambiamento della forma della frontiera a rendimenti variabili $\left(S T E C H_{t, t+k}\right)$, ovvero dall'allontanamento (valori maggiori di uno) o avvicinamento (valori minori di uno) rispetto alla frontiera a rendimenti costanti. Formalmente $T E C H_{t, t+k}$ può dunque essere scomposto come segue (Wheelock e Wilson, 1999):

$T E C H_{t, t+k}=\left(\frac{D_{o}^{t}\left(\mathbf{x}^{t+k}, \mathbf{y}^{t+k} \mid V R S\right)}{D_{o}^{t+k}\left(\mathbf{x}^{t+k}, \mathbf{y}^{t+k} \mid V R S\right)} \times \frac{D_{o}^{t}\left(\mathbf{x}^{t}, \mathbf{y}^{t} \mid V R S\right)}{D_{o}^{t+k}\left(\mathbf{x}^{t}, \mathbf{y}^{t} \mid V R S\right)}\right)^{1 / 2} \times$

$\times\left(\frac{D_{o}^{t}\left(\mathbf{x}^{t+k}, \mathbf{y}^{t+k} \mid C R S\right) / D_{o}^{t}\left(\mathbf{x}^{t+k}, \mathbf{y}^{t+k} \mid V R S\right.}{D_{o}^{t+k}\left(\mathbf{x}^{t+k}, \mathbf{y}^{t+k} \mid C R S\right) / D_{o}^{t+k}\left(\mathbf{x}^{t+k}, \mathbf{y}^{t+k} \mid V R S\right)} \times \frac{D_{o}^{t}\left(\mathbf{x}^{t}, \mathbf{y}^{t} \mid C R S\right) / D_{o}^{t}\left(\mathbf{x}^{t}, \mathbf{y}^{t} \mid V R S\right.}{D_{o}^{t+k}\left(\mathbf{x}^{t}, \mathbf{y}^{t} \mid C R S\right) / D_{o}^{t+k}\left(\mathbf{x}^{t}, \mathbf{y}^{t} \mid V R S\right)}\right)^{1 / 2}=$

$=\mathrm{PTECH}_{t, t+k} \times \mathrm{STECH}_{t, t+k}$

Infine, la terza scomposizione impiegata separa, nella misura del cambiamento tecnologico $\left(\mathrm{TECH}_{t, t+k}\right)$, una componente che non dipende dal mutamento di composizione di input e/o output utilizzati e prodotti dall'impresa (disembodied technical change), da una che dipende, invece, dalla composizione di input e/o output (embodied technical change). Il cambiamento tecnologico può essere suddiviso, quindi, in effetto di magnitudine, $M A T E C H_{t, t+k}$ (MAgnitude TEchnical CHange) ed effetto di composizione degli input, IBTE$\mathrm{CH}_{t, t+k}$ (Input Biased TEchnical CHange). ${ }^{5}$ Pertanto, la componente $T E C H_{t, t+k}$ può essere riscritta come segue (Färe e Grosskopf, 1996):

$T E C H_{t, l+k}=\frac{D_{o}^{t}\left(\mathbf{x}^{t}, \mathbf{y}^{t} \mid C R S\right)}{D_{o}^{t+k}\left(\mathbf{x}^{t+k}, \mathbf{y}^{t+k} \mid C R S\right)} \times\left[\frac{D_{o}^{t+k}\left(\mathbf{x}^{t}, \mathbf{y}^{t} \mid C R S\right)}{D_{o}^{t}\left(\mathbf{x}^{t}, \mathbf{y}^{t} \mid C R S\right)} \times \frac{D_{o}^{t}\left(\mathbf{x}^{t+k}, \mathbf{y}^{t} \mid C R S\right)}{D_{o}^{t+k}\left(\mathbf{x}^{t+k}, \mathbf{y}^{t} \mid C R S\right)}\right]^{1 / 2}=M A T E C H_{t, t+k} \times I^{\prime}$ IBTECH ${ }_{t, t+k}$ [7]

L'effetto di magnitudine $\left(M^{\prime} A T E C H_{t, t+k}\right)$ cattura il progresso tecnico definito Hicks-neutrale, nel senso che il cambiamento tecnologico avviene lasciando immutata la composizione relativa dei fattori: data la stessa combinazione e quantità di input otteniamo lo stesso paniere di output ma di volume differente. Se l'effetto magnitudine è superiore a uno significa che con la stessa com-

5. Non teniamo conto qui di una terza componente, l'effetto di composizione degli output, perché nel nostro lavoro consideriamo sempre una funzione di produzione con un singolo output. 
binazione e quantità di input si ottiene un output maggiore in termini di volume, viceversa se è inferiore a uno si ottiene un volume di output minore. $I B$ $\mathrm{TECH}_{t, t+k}$ invece cattura gli effetti di composizione degli input: il cambiamento tecnologico in questo caso può essere associato a un unico fattore, o comportare un cambiamento di composizione dei fattori produttivi. Nel caso in cui tale componente assuma valori minori di uno allora sono presenti effetti di composizione negativi, viceversa se tale componente assume valori superiori a uno siamo in presenza di effetti di composizione positivi.

L'indice di Malmquist, e ognuna delle scomposizioni presentate, si ottengono dunque dalla combinazione di più funzioni di distanza, calcolate rispetto a frontiere di produzione definite sotto diverse ipotesi. Empiricamente, le funzioni di distanza possono essere stimate mediante l'utilizzo di tecniche non parametriche DEA. In particolare, mediante la DEA si stima la distanza dell'impresa $i$ dalla sua proiezione efficiente sulla frontiera, individuata come combinazione convessa dei vettori input/output delle imprese efficienti osservate. Nel caso di rendimenti di scala costanti, dato un campione di $N$ imprese, la distanza dell'impresa $i$ è ottenuta risolvendo il seguente programma lineare (Charnes, Cooper e Rhodes, 1978):

$\left[D_{o}^{p}\left(\mathbf{y}_{i}^{q}, \mathbf{x}_{i}^{q} \mid C R S\right)\right]^{-1}=\max _{\varphi}\left\{\sum_{j=1}^{N} \lambda_{j} y_{j m}^{p} \geq \varphi y_{i m}^{q}, m=1, \ldots, M ; \sum_{j=1}^{N} \lambda_{j} x_{j s}^{p} \leq x_{i s}^{q}, s=1, \ldots, S ; \lambda \in \Re_{+}^{N}\right\}$

dove $p, q=t, t+k$. Il vettore $\lambda$ è composto dai pesi assegnati alle imprese nella costruzione della combinazione convessa che rappresenta la proiezione efficiente dell'impresa $i$. Nel caso di rendimenti di scala variabili, invece, la distanza dell'impresa $i$ dalla frontiera è ottenuta come soluzione di un programma lineare simile a [8] in cui è aggiunto un ulteriore vincolo sul vettore $\lambda$ (Banker, Charnes e Cooper, 1984):

$\left[D_{o}^{p}\left(\mathbf{y}_{i}^{q}, \mathbf{x}_{i}^{q} \mid V R S\right)\right]^{-1}=\max _{\varphi}\left\{\sum_{j=1}^{N} \lambda_{j} y_{j m}^{p} \geq \varphi y_{i m}^{q}, m=1, \ldots, M ; \sum_{j=1}^{N} \lambda_{j} x_{j s}^{p} \leq x_{i s}^{q}, s=1, \ldots, S ; \sum_{j=1}^{N} \lambda_{j}=1, \lambda \in \Re_{+}^{N}\right\}$

\subsection{Analisi degli "outlier"}

L'approccio utilizzato per la stima delle frontiere ha una natura prettamente deterministica e le frontiere sono individuate dalle osservazioni estreme del campione analizzato e dalle loro combinazioni lineari. È chiaro, dunque, come la presenza di osservazioni "outlier" possa avere un impatto fortemente distorsivo. Nell'individuazione degli "outlier" in dataset di dimensioni considerevoli si è in presenza però di due problemi: l'estrema difficoltà a ispezionare "manualmente" il dataset e l'efficienza computazionale delle procedure da implementare per la ricerca degli "outlier". 
Per superare tali difficoltà, nel presente lavoro si è utilizzata una tecnica proposta da Sampaio de Sousa e Stosic (2005). La tecnica in questione individua le imprese che "condizionano maggiormente" le misure di efficienza delle altre imprese. In particolare, dato un insieme di $N$ osservazioni, l'approccio proposto si basa sul concetto di "leverage" ovvero, per ogni impresa $i$, l'effetto prodotto sulle efficienze calcolate per altre $N$ - 1 imprese quando l'impresa $i$ è eliminata dal campione osservato. Il "leverage" dell'impresa $i$ è quindi calcolato come segue:

leverage $_{i}=\sqrt{\frac{\sum_{j=1 ; j \neq i}^{N}\left(\theta_{j i}^{*}-\theta_{j}\right)^{2}}{N-1}}$

dove $\theta_{j}$ è l'efficienza (o distanza) rispetto alla frontiera calcolata per l'impresa $j$ considerando tutte le $N$ imprese, mentre $\theta_{j i}^{*}$ è l'efficienza per l'impresa $j$ quando l'impresa $i$ è eliminata dal dataset. Per superare i problemi computazionali, la procedura proposta da Sampaio de Sousa e Stosic (2005) combina bootstrap e jackknife resampling: si estrae un sottoinsieme di imprese dall'insieme di imprese complessivo e si calcola il "leverage" di ogni impresa del sottoinsieme estratto; queste operazioni sono, quindi, ripetute un numero sufficiente di volte ottenendo alla fine un "leverage" medio per ogni impresa.

L'idea alla base della procedura utilizzata è che le imprese "outlier" presentino un "leverage" che supera un certo valore limite. Una soluzione proposta in letteratura consiste nell'individuare il valore limite come il "leverage" medio $\bar{l}$ (calcolato come media dei "leverage" delle $N$ imprese) moltiplicato per il logaritmo della cardinalità dell'insieme delle imprese analizzato: $\bar{l} \log (N)$.

Un problema non secondario è però legato al trade-off tra necessità di eliminare osservazioni anomale e quella di non perdere troppe osservazioni. Pertanto nel presente lavoro sono state classificate come "outlier" imprese il cui "leverage" superava un limite più lasco, ovvero $2 \bar{l} \log (N)$.

L'analisi è stata eseguita per ogni anno per ogni settore. ${ }^{6}$ In ogni settore sono state eliminate le imprese che in almeno un anno evidenziavano un "leverage" superiore alla soglia limite calcolata per lo specifico settore di appartenenza, nello specifico anno. Dopo l'eliminazione degli "outlier", il panel bilanciato si riduce a settemila e trecentoquarantasette imprese che costituiscono la base della nostra analisi.

\section{La dinamica della produttività nelle imprese manifatturiere italiane}

In questo paragrafo riportiamo i risultati riguardanti la misurazione e scomposizione della produttività totale dei fattori relativi al campione di imprese ana-

6. Le efficienze (o distanze) per il calcolo del "leverage" sono state ottenute assumendo rendimenti di scala costanti. 
lizzato. Per ogni impresa, dopo avere calcolato le distanze dalle frontiere intertemporali stimate per il settore di appartenenza e ottenuti gli indici di Malmquist e le scomposizioni proposte, si sono calcolate le variazioni percentuali medie annue del Malmquist e sue componenti. ${ }^{7}$ Si sono calcolati poi i tassi medi annui pesati delle variazioni a livello settoriale. ${ }^{8}$ La relazione tra i valori delle scomposizioni ottenute - tra i quali sussiste una relazione moltiplicativa a livello di singola impresa - assume, con un certo grado di approssimazione, una forma additiva se presentata in termini di tassi medi annui di variazione aggregati. ${ }^{9}$ Un segno negativo del tasso medio annuo di crescita di un indice corrisponde quindi a valori inferiori a uno nel periodo analizzato, mentre un segno positivo corrisponde a valori maggiori di uno.

\subsection{Efficienza e cambiamento tecnologico}

Il primo esercizio che proponiamo è un confronto dei tassi medi annui di variazione dell'indice di Malmquist per le sottosezioni della manifattura (come definite dalla classificazione ATECO-2002) per il periodo 1996-2006 (tab. 3). Questo indice ci offre un'idea dell'andamento della produttività totale dei fattori nei vari comparti della manifattura nel decennio in esame. Alcune osservazioni sono immediate. Anzitutto si conferma, anche a livello disaggregato, la modesta dinamica della produttività che si mantiene, con l'eccezione di pochi settori, come il meccanico e la produzione di apparecchi elettrici, su tassi di crescita largamente inferiori all' $1 \%$ medio annuo. Nella gran parte dei settori, la crescita della grandezza in esame è anche inferiore allo $0,5 \% \mathrm{e}$ in qualche settore risulta negativa. Inoltre, va notato che le due componenti della

7. Va notato che per alcune imprese non tutti i programmi lineari necessari a calcolare le distanze per la scomposizione dell'indice di Malmquist restituiscono soluzioni ammissibili. Questo fatto può accadere, in particolare, nella scomposizione della componente tecnologica. Per questo motivo in alcuni casi la ricomposizione delle componenti isolate non è uguale al valore originale. Nei casi in cui la numerosità delle soluzioni non ammissibili è particolarmente alta, si è preferito omettere il dato. 8. Il peso assegnato a ogni impresa nelle aggregazioni è ottenuto come media nel periodo $t, t+k$ del rapporto dell'output (ricavi delle vendite) della singola impresa sull'output totale del settore.

9. L'approssimazione dipende sia dall'effetto dell'aggregazione ponderata, ma anche dalla presenza di termini congiunti. Per esempio, se calcolata per la singola impresa, la crescita media annua in un certo periodo del Malmquist è esattamente pari alla variazione media annua di $E F F C H$ più la variazione di $T E C H$ più una componente residuale data dalla variazione di $E F F C H \times T E C H$. Lo stesso tipo di ragionamento vale anche per le ulteriori scomposizioni delle componenti del Malmquist. 
variazione dell'efficienza e del mutamento tecnologico agiscono in modo piuttosto variegato nel determinare l'andamento della produttività. Si trovano tanto settori in cui la tecnologia migliora, ma c'è un arretramento dell'efficienza, come il tessile, il cuoio, la metallurgia o la produzione di apparecchi elettrici, quanto settori in cui accade il contrario, come nel settore cartario e nella chimica e infine si hanno settori in cui i segni di entrambe le componenti sono positive, come il meccanico e i mezzi di trasporto.

Tab. 3 - Indice di Malmquist (MALM) e sua scomposizione in cambiamento tecnologico (TECH) e variazione di efficienza (EFFCH) per il periodo 1996-2006. (Variazioni medie annue \%)

\begin{tabular}{lrrr}
\hline Settore & & $1996-2006$ & \\
& MALM & EFFCH & TECH \\
\hline Alimentare e tabacco & 1,069 & 0,050 & 1,018 \\
Tessile e abbigliamento & 0,353 & $-0,221$ & 0,574 \\
Cuoio & $-0,004$ & $-0,171$ & 0,167 \\
Legno & 0,268 & 0,226 & 0,043 \\
Carta, stampa e editoria & 0,090 & 0,534 & $-0,442$ \\
Raffinerie di petrolio & $-1,004$ & $-0,685$ & $-0,326$ \\
Chimica & $-0,032$ & 0,147 & $-0,180$ \\
Gomma e plastica & 0,490 & 0,194 & 0,296 \\
Minerali non metalliferi & 0,068 & 0,041 & 0,030 \\
Metallo e prodotti in metallo & 0,273 & $-0,033$ & 0,307 \\
Macchine e apparecchi meccanici & 1,019 & 0,143 & 0,873 \\
Apparati elettrici e di precisione & 1,224 & $-0,120$ & 1,346 \\
Mezzi di trasporto & 0,558 & 0,169 & 0,387 \\
Altre industrie manifatturiere & 0,196 & $-0,175$ & 0,372 \\
\hline
\end{tabular}

Fonte: nostre elaborazioni su dati SdC19962006.

Interessante è nondimeno approfondire l'analisi degli andamenti segnalati scomponendo in sottoperiodi la finestra temporale a disposizione. La tab. 4 illustra l'andamento dell'indice di Malmquist e delle sue componenti di variazione di efficienza e cambiamento tecnologico in tre sottoperiodi: 1996-2000, 2000-2003, 2003-2006.

I tre sottoperiodi colgono la fase di ingresso nell'euro (1999), il ciclo negativo centrato sul 2001 e il periodo precedente la crisi tuttora in corso. Alcune evidenze sono immediate. In primo luogo, si nota come i peggiori risultati in termini di crescita della produttività si presentino nel primo periodo, quello a cavallo dell'ingresso nell'euro in cui si osserva una variazione negativa della produttività per quasi tutti i settori. 
E. Tundis, E. Zaninotto, R. Gabriele, S. Trento Produttività delle imprese italiane: 1996-2006

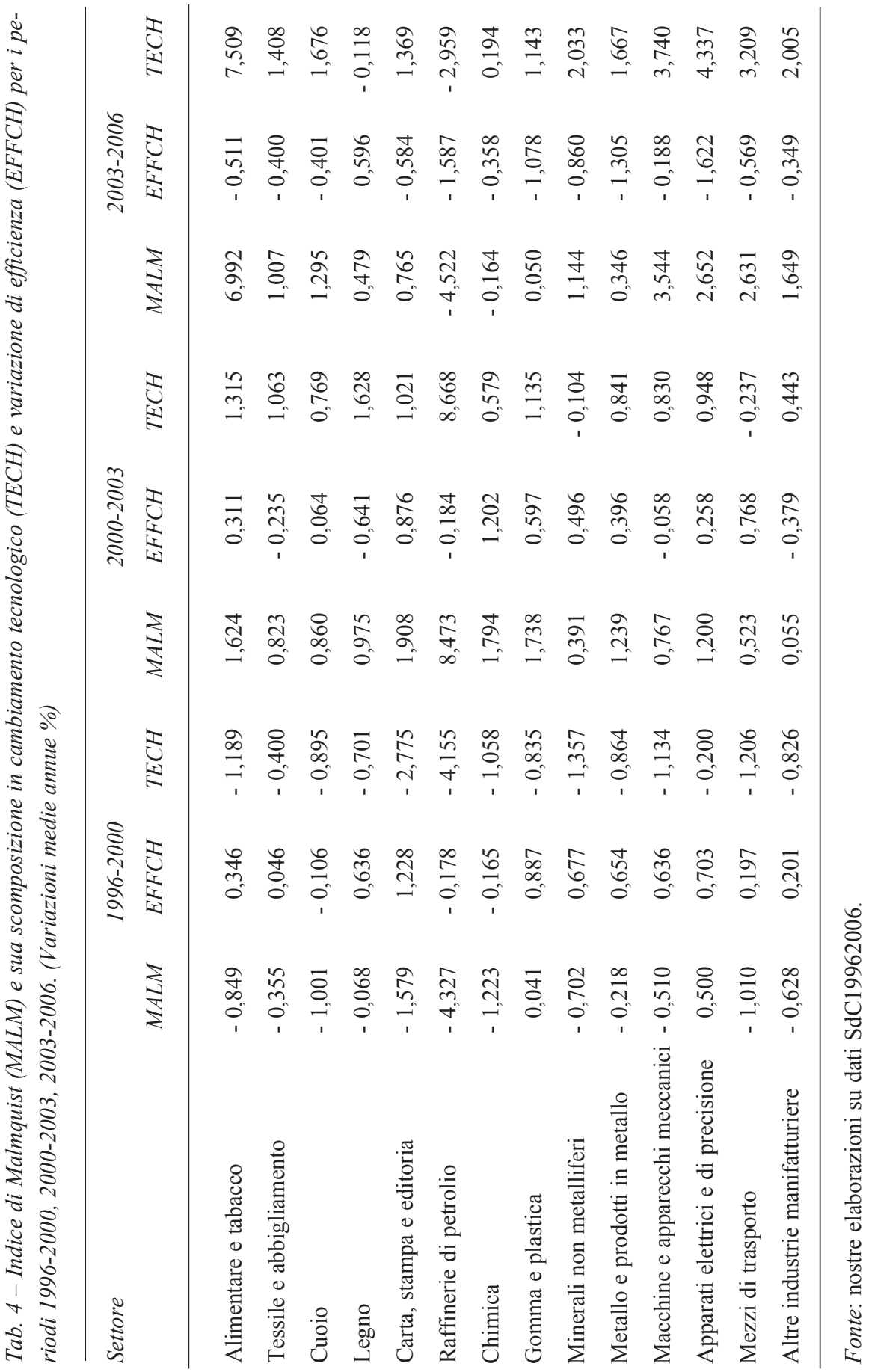


Il periodo intermedio presenta una ripresa generalizzata della produttività, mentre quello finale mostra segni sia positivi sia negativi, con una larga prevalenza dei primi, ma in generale di dimensione inferiore a quelli del periodo 2000-2003.

Un secondo elemento che emerge con chiarezza è dato dal peso delle due componenti della produttività nei diversi periodi. Nel primo periodo si osserva, in modo uniforme in tutti i settori, una sorta di "retrocessione tecnologica": il segno negativo del valore medio di TECH segnala infatti che la frontiera produttiva nel periodo si sposta verso il basso.

Questo consistente spostamento è in parte compensato da un recupero di efficienza, il cui coefficiente è quasi ovunque positivo. ${ }^{10}$

Nel secondo periodo (2000-2003) la crescita della produttività è sostenuta in tutti i settori ed è trascinata principalmente dal cambiamento tecnologico che in qualche caso si rafforza con un miglioramento dell'efficienza, ma la coincidenza con il ciclo consiglia di tenere conto con cautela questo dato, data la particolare sensibilità delle osservazioni ai punti iniziali e finali del ciclo. Più chiaro è l'andamento che si presenta nel periodo 2003-2006, all'uscita dalla crisi, quando la situazione diventa più varia dal punto di vista delle performance produttive globali, piuttosto diverse tra settori, mentre presenta un pattern uniforme dal punto di vista delle due componenti dell'indice di Malmquist.

Più precisamente, si è ora in presenza quasi ovunque di tendenze contrastanti dovute alla componente tecnologica e a quella di efficienza, con segni positivi della prima e negativi della seconda.

Questa evidenza segnala che vi è un processo di avanzamento tecnologico, dovuto alle migliori imprese che determinano la posizione della frontiera, a fronte del quale sta un ampliamento della distribuzione delle imprese al di sotto della frontiera di produzione.

È nondimeno l'abbassamento dell'efficienza media che desta maggiori preoccupazioni e difficoltà interpretative. L'andamento è preoccupante perché segnala che in un periodo di relativa ripresa e ristrutturazione produttiva molte imprese non stanno al passo e arretrano rispetto alla frontiera.

La fig. 1, chiarisce la dinamica appena descritta riportando l'evoluzione nel tempo della densità di probabilità delle efficienze nel caso particolare della produzione di apparecchi elettronici nei periodi 1996-2000 e 2003-2006.

10. La retrocessione tecnologica da noi segnalata alla fine degli anni 1990 può trovare una parziale conferma in Milana, Nascia e Zeli (2008), che tuttavia si riferiscono al periodo 1998-2004: la nostra periodizzazione ci sembra invece che possa dare conto in modo più preciso dei diversi andamenti nel tempo. 
E. Tundis, E. Zaninotto, R. Gabriele, S. Trento Produttività delle imprese italiane: 1996-2006

Fig. 1 - Kernel density estimation dell'efficienza nel settore "apparati elettrici e di precisione"
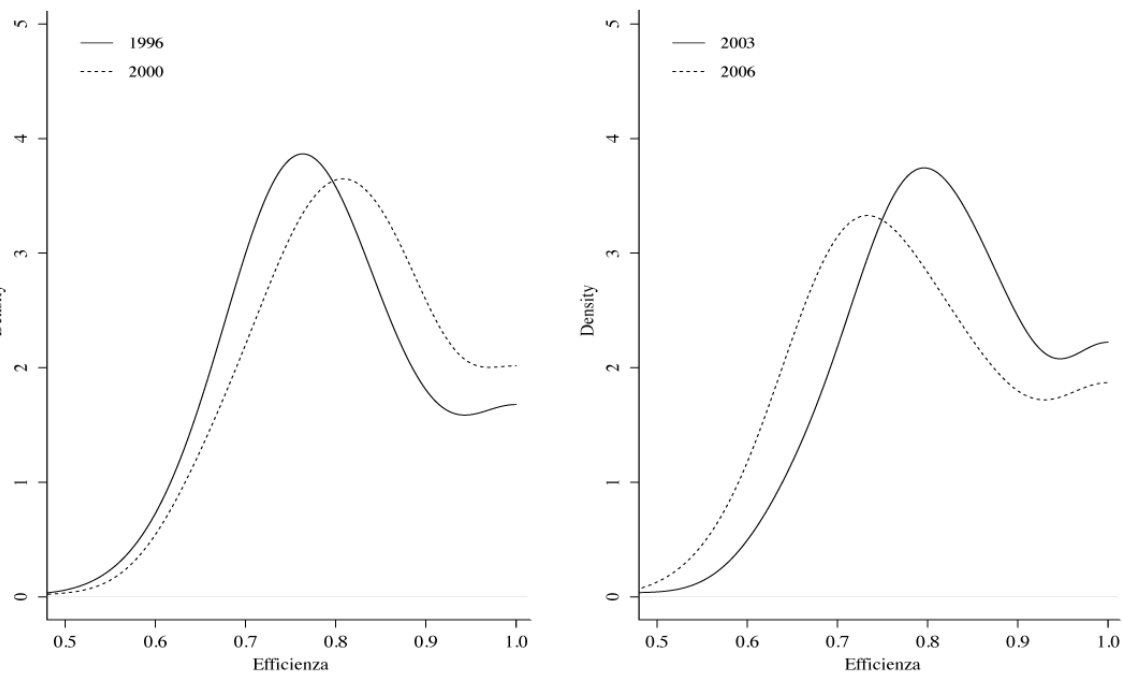

Fonte: nostre elaborazioni su dati SdC19962006.

Fig. 2 - Dispersione dei settori manifatturieri per cambiamento tecnologico (TECH) e variazione di efficienza (EFFCH). Periodi 1996-2000 e 2003-2006

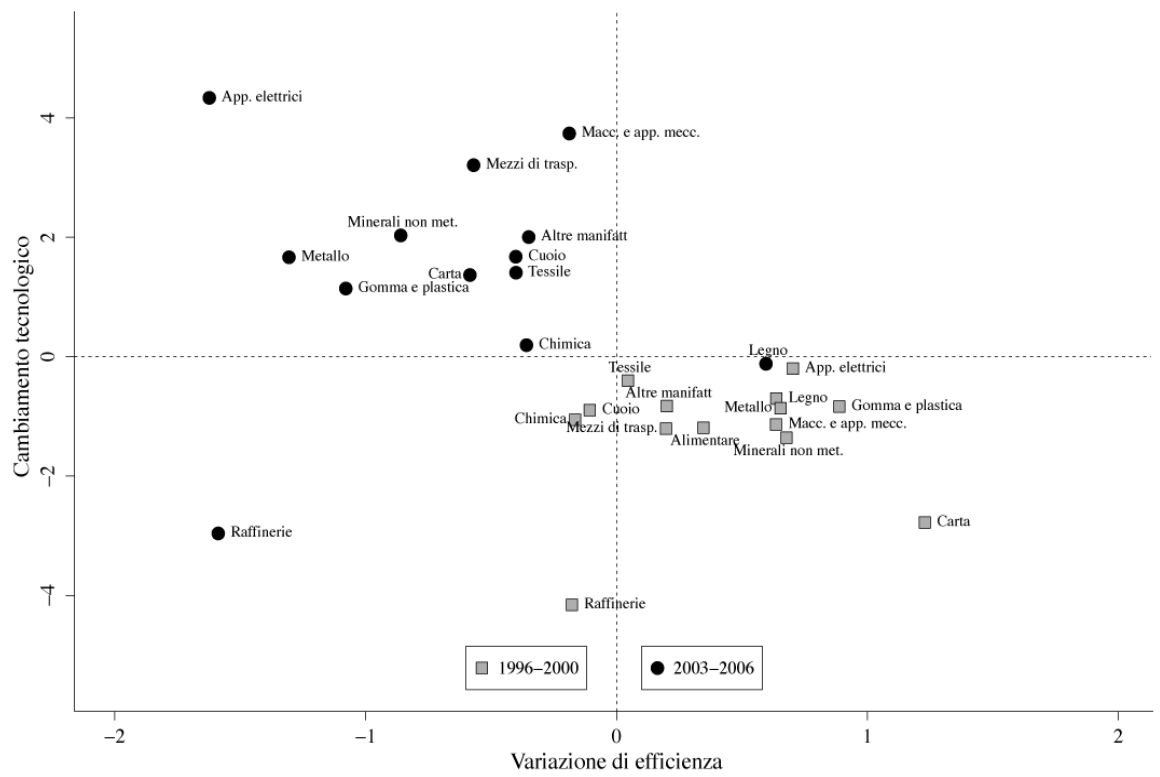

Fonte: nostre elaborazioni su dati SdC19962006. 
Si nota chiaramente come alla fine del primo periodo (anno 2000) la distribuzione si sia spostata verso destra indicando un aumento di efficienza generalizzato a gran parte delle imprese del settore e in particolare il raggiungimento di condizioni prossime alla piena efficienza per un maggiore numero di imprese rispetto all'inizio del periodo (anno 1996). L'evoluzione cambia drasticamente nell'ultimo periodo (2003-2006), in cui lo spostamento verso sinistra della distribuzione indica una diffusa perdita di efficienza, in particolare delle imprese che stavano sotto la frontiera, dovuta all'accelerazione di una parte delle imprese già efficienti che hanno spostato la frontiera produttiva.

L'aumento della dispersione risulta altresì dal grafico sulla modificazione nel tempo della variazione dell'efficienza e del cambiamento tecnologico. Nella fig. 2 presentiamo la situazione nei vari settori nei due periodi estremi: è evidente lo spostamento dell'insieme dei settori dal quadrante sud ovest a quello nord est della mappa, con la generalizzata crescita del cambiamento tecnologico a fronte di una riduzione di efficienza.

\subsection{I fattori di scala e di composizione degli input}

La tab. 5 scompone la variazione di efficienza nella componente di pura efficienza e di efficienza di scala nei tre sottoperiodi. L'evidenza più chiara è che nel primo periodo (1996-2000) le imprese tentano un recupero di efficienza, anche in assenza di avanzamento tecnologico, che risulta contemporaneamente da una riduzione delle inefficienze e un recupero di scala. Viceversa, nei periodi successivi, andamenti analoghi non sono altrettanto chiari e pervasivi.

Tab. 5 - Scomposizione della variazione di efficienza (EFFCH) nelle componenti di PEFCH e SCH per i periodi 1996-2000, 2000-2003 e 2003-2006. (Variazioni medie annue \%)

\begin{tabular}{|c|c|c|c|c|c|c|c|c|c|}
\hline \multirow[t]{2}{*}{ Settore } & \multicolumn{3}{|c|}{$1996-2000$} & \multicolumn{3}{|c|}{$2000-2003$} & \multicolumn{3}{|c|}{$2003-2006$} \\
\hline & $\mathrm{EFFCH}$ & I PEFCH & $\mathrm{I} \mathrm{SCH}$ & $E F F C H$ & PEFCH & $\mathrm{ISCH}$ & EFFCH & PEFCH & $\mathrm{SCH}$ \\
\hline Alimentare e tabacco & 0,346 & 0,520 & $-0,170$ & 311 & 0,216 & 0,100 & $-0,511$ & $-0,412$ & $-0,096$ \\
\hline Tessile e abbigliamento & 0,046 & $-0,215$ & 0,268 & $-0,235$ & $-0,129$ & $-0,100$ & $-0,400$ & $-0,198$ & $-0,189$ \\
\hline Cuoio & $-0,106$ & $-0,095$ & $-0,009$ & 0,064 & 0,122 & $-0,052$ & $-0,401$ & $-0,486$ & 0,088 \\
\hline Legno & 0,636 & 0,280 & 0,357 & $-0,641$ & $-0,314$ & $-0,323$ & 0,596 & 0,336 & 0,264 \\
\hline Carta, stampa e editoria & 1,228 & 0,841 & 0,390 & 0,876 & 0,946 & $-0,057$ & $-0,584$ & $-0,707$ & 0,132 \\
\hline Raffinerie di petrolio & $-0,178$ & $-0,069$ & $-0,108$ & $-0,184$ & 0,202 & $-0,385$ & $-1,587$ & $-0,208$ & $-1,382$ \\
\hline Chimica & $-0,165$ & $-0,202$ & 0,040 & 1,202 & 0,218 & 0,991 & $-0,358$ & 0,194 & $-0,549$ \\
\hline Gomma e plastica & 0,887 & 0,591 & 0,303 & 0,597 & 0,168 & 0,432 & $-1,078$ & $-0,514$ & $-0,562$ \\
\hline Minerali non metalliferi & i 0,677 & 0,545 & 0,133 & 0,496 & $-0,021$ & 0,522 & $-0,860$ & $-0,857$ & 0,000 \\
\hline $\begin{array}{l}\text { Metallo e prodotti in } \\
\text { metallo }\end{array}$ & 0,654 & 0,011 & 0,646 & 0,396 & 0,186 & 0,216 & $-1,305$ & $-0,451$ & $-0,854$ \\
\hline Macchine e app. mecc. & 0,636 & 0,118 & 0,521 & $-0,058$ & 0,299 & $-0,352$ & $-0,188$ & $-1,025$ & 0,865 \\
\hline $\begin{array}{l}\text { App. elettrici e di } \\
\text { precisione }\end{array}$ & 0,703 & 0,354 & 0,361 & 0,258 & $-0,578$ & 0,850 & $-1,622$ & $-1,046$ & $-0,580$ \\
\hline Mezzi di trasporto & 0,197 & 0,124 & 0,084 & 0,768 & 0,538 & 0,235 & $-0,569$ & $-0,150$ & $-0,421$ \\
\hline $\begin{array}{c}\text { Altre industrie } \\
\text { manifatturiere }\end{array}$ & 0,201 & 0,071 & 0,134 & $-0,379$ & 0,316 & $-0,686$ & $-0,349$ & $-0,195$ & $-0,149$ \\
\hline
\end{tabular}

Fonte: nostre elaborazioni su dati SdC19962006. 
Tra il 2003 e il 2006 l'efficienza media, come si è detto, si abbassa nonostante un avanzamento della frontiera tecnologica: segno questo di una maggiore dispersione delle imprese rispetto alla frontiera tecnologica e della persistenza stabile di imprese che accumulano ritardi tecnologici. Di fronte a questo fenomeno, non si assiste neppure a un chiaro recupero in termini di dimensionamento ottimale delle imprese, a differenza di quanto avvenne tra il 1996 e il 2000. Ancora una volta, sembra di essere in presenza di due storie diverse. Questa ipotesi trova una conferma anche nell'osservazione della dimensione media delle imprese. La dimensione media, in termini di addetti, presenta un salto tra il 1996 e il 2000 (tab. 6), sostanzialmente generalizzato in tutti i settori, mentre successivamente la dimensione si stabilizza. La dimensione, espressa in termini di fatturato medio a valori costanti, manifesta un salto analogo, seppure meno evidente.

Tab. 6-Addetti per settore e anno

Settore

Anno

$1996 \quad 2000 \quad 2003 \quad 2006$

Alimentare e tabacco

Media

Dev. std.

Tessile e abbigliamento

Cuoio

Legno

Carta, stampa e editoria

Raffinerie di petrolio

Chimica

Gomma e plastica

Minerali non metalliferi

Metallo e prodotti in metallo

Macchine e app. meccanici

App. elettrici e di precisione

Mezzi di trasporto

Altre industrie manifatturiere
Media

Dev. std.

Media

Dev. std.

Media

Dev. std.

Media

Dev. std.

Media

Dev. std.

Media

Dev. std.

Media

Media

Dev. std.

Media

Dev. std.

Media

Dev. std.

Media

Dev. std.

Media

Dev. std.

Media

Dev. std.
Dev. std.
40,03

45,30

53,62

64,86

52,45

59,27

39,88

34,10

41,16

39,03

43,27

66,53

49,95

74,14

46,12

50,17

44,46

48,68

46,26

48,68

49,30

51,75

46,35

42,92

60,47

70,66

45,67

38,33

\section{4,87}

53,40

57,05

67,58

54,74

61,22

45,10

41,07

44,87

40,35

44,09

60,07

56,52

80,73

54,81

62,59

49,43

54,80

53,83

56,34

55,37

56,71

54,10

51,34

67,21

74,55

52,67

44,25
46,35

56,22

53,91

64,73

50,37

53,97

43,57

39,80

44,03

39,18

47,14

60,57

56,40

76,81

54,17

65,02

49,79

57,30

53,42

58,58

55,08

59,94

52,73

52,17

63,57

67,01

51,23

46,28
44,31

55,56

50,33

65,79

45,70

48,80

43,38

40,97

43,94

40,79

51,24

70,51

59,50

75,74

54,77

71,06

49,65

59,21

54,73

63,06

56,81

66,52

52,98

52,34

63,88

70,80

49,52

49,03

Fonte: nostre elaborazioni su dati SdC19962006. 
E. Tundis, E. Zaninotto, R. Gabriele, S. Trento Productivity of Italian firms: 1996-2006

Per il totale delle imprese manifatturiere, il fatturato medio espresso a prezzi costanti passa da sei virgola nove milioni di euro nel 1996, a otto virgola cinque milioni di euro nel 2000, per stabilizzarsi a otto virgola sei milioni di euro nel 2003 e crescere nell'ultimo periodo per arrivare a nove virgola otto milioni di euro nel 2006.

La scomposizione della componente tecnologica in tab. 7 mostra che tanto l'arretramento della frontiera del primo periodo, che il suo avanzamento nel terzo sono dovuti alla componente tecnologica pura, ovvero a uno spostamento della frontiera a rendimenti variabili di scala.

Al tempo stesso la componente $S T E C H_{t, t+k}$ assume con poche eccezioni valori ovunque negativi. Un valore negativo del tasso di crescita medio annuo dell'indice indica che la frontiera a rendimenti variabili si avvicina a quella a rendimenti constanti. Questo andamento è coerente con l'idea che lungo tutto il periodo in esame sia aumentata nel sistema la flessibilità di volume. Infine, la tab. 8 mostra chiaramente che la "retrocessione" della frontiera osservata nel periodo 1996-2000 è dovuta principalmente a effetti di magnitudine Hicks-neutrali, solo parzialmente compensati da un miglioramento della composizione dei fattori.

Nei due periodi successivi, invece, lo spostamento della frontiera dipende congiuntamente dai due effetti.

Tab. 7 - Scomposizione del cambiamento tecnologico (TECH) nelle componenti PTECH e STECH per i periodi 1996-2000, 2000-2003 e 2003-2006. (Variazioni medie annue \%)

Settore 1996-2000 2000-2003 2003-2006

TECH PTECH STECH TECH PTECH STECH TECH PTECH STECH

\begin{tabular}{lrrrrrrrrr}
\hline Alimentare e tabacco & $-1,189$ & $-1,042$ & $-0,096$ & 1,31 & 1,865 & $-0,490$ & 7,509 & - & - \\
Tessile e abbigliamento & $-0,400$ & 0,171 & $-0,492$ & 1,06 & 0,557 & 0,516 & 1,408 & 1,604 & $-0,181$ \\
Cuoio & $-0,895$ & $-0,997$ & 0,144 & 0,77 & $-0,746$ & 0,740 & 1,676 & 1,642 & $-0,004$ \\
Legno & $-0,701$ & 0,108 & $-0,742$ & 1,63 & 1,004 & 0,511 & $-0,118$ & 0,873 & $-0,958$ \\
Carta, stampa e editoria & $-2,775$ & $-1,755$ & $-0,814$ & 1,02 & 0,789 & 0,172 & 1,369 & 2,142 & $-0,727$ \\
Raffinerie di petrolio & $-4,155$ & $-5,022$ & 1,037 & 8,67 & 8,555 & $-0,031$ & $-2,959$ & $-4,046$ & 1,120 \\
Chimica & $-1,058$ & $-0,134$ & $-0,638$ & 0,58 & 1,631 & $-1,018$ & 0,194 & $-0,350$ & 0,610 \\
Gomma e plastica & $-0,835$ & 0,174 & $-0,924$ & 1,13 & 1,420 & $-0,262$ & 1,143 & 1,202 & $-0,005$ \\
Minerali non metalliferi & $-1,357$ & $-0,735$ & $-0,530$ & $-0,10$ & 0,353 & $-0,432$ & 2,033 & 2,716 & $-0,650$ \\
Metallo e prodotti in & $-0,864$ & 0,011 & $-0,850$ & 0,84 & 1,052 & $-0,252$ & 1,667 & 1,152 & 0,560 \\
metallo & $-1,134$ & $-0,415$ & $-0,680$ & 0,83 & 0,552 & 0,284 & 3,740 & 5,095 & $-1,308$ \\
$\begin{array}{l}\text { Macchine e app. mecc. } \\
\text { Apparati elettrici e di }\end{array}$ & $-0,200$ & 0,537 & $-0,681$ & 0,95 & 2,015 & $-1,119$ & 4,337 & 4,465 & $-0,027$ \\
precisione & $-1,206$ & 0,138 & $-1,096$ & $-0,24$ & $-0,017$ & $-0,214$ & 3,209 & 3,222 & $-0,054$ \\
$\begin{array}{l}\text { Mezzi di trasporto } \\
\text { Altre industrie }\end{array}$ & $-0,826$ & $-0,413$ & $-0,357$ & 0,44 & $-0,161$ & 0,614 & 2,005 & 2,018 & $-0,050$ \\
manifatturiere & & & & & & & &
\end{tabular}

Fonte: nostre elaborazioni su dati SdC19962006. 
E. Tundis, E. Zaninotto, R. Gabriele, S. Trento Produttività delle imprese italiane: 1996-2006

Tab. 8 - Scomposizione del cambiamento tecnologico (TECH) nelle componenti MATECH e IBTECH per i periodi 1996-2000, 2000-2003 e 2003-2006. (Variazioni medie annue \%)

Settore

1996-2000

2000-2003

2003-2006

TECH MATECH IBTECH TECH MATECH IBTECH TECH MATECH IBTECH

\begin{tabular}{|c|c|c|c|c|c|c|c|c|c|}
\hline Alimentare e tabacco & $-1,189$ & $-1,558$ & 0,406 & 1,315 & 0,380 & 0,958 & 7,509 & $-0,594$ & 9,473 \\
\hline Tessile e abbigliamento & $-0,400$ & $-0,645$ & 0,270 & 1,063 & 0,897 & 0,183 & 1,408 & 1,148 & 0,266 \\
\hline Cuoio & $-0,895$ & $-1,213$ & 0,348 & 0,769 & $-0,252$ & 1,045 & 1,676 & 1,236 & 0,780 \\
\hline Legno & $-0,701$ & $-1,133$ & 0,461 & 1,628 & 1,004 & 0,641 & $-0,118$ & $-0,657$ & 0,577 \\
\hline Carta, stampa e editoria & $-2,775$ & $-3,061$ & 0,329 & 1,021 & 0,689 & 0,353 & 1,369 & 1,071 & 0,353 \\
\hline Raffinerie di petrolio & $-4,155$ & - 11,724 & 9,503 & 8,668 & 4,349 & 4,129 & $-2,959$ & $-7,801$ & 5,914 \\
\hline Chimica & $-1,058$ & $-1,842$ & 0,958 & 0,579 & 0,311 & 0,279 & 0,194 & $-0,231$ & 0,449 \\
\hline Gomma e plastica & $-0,835$ & $-1,071$ & 0,252 & 1,135 & 0,918 & 0,237 & 1,143 & 0,918 & 0,236 \\
\hline Minerali non metalliferi & $-1,357$ & $-1,790$ & 0,458 & 0,104 & $-0,380$ & 0,283 & 2,033 & 1,774 & 0,266 \\
\hline $\begin{array}{l}\text { Metallo e prodotti in } \\
\text { metallo }\end{array}$ & $-0,864$ & $-1,315$ & 0,479 & 0,841 & 0,589 & 0,265 & 1,667 & 0,290 & 1,421 \\
\hline Macchine e app. mecc. & $-1,134$ & $-1,343$ & 0,218 & 0,830 & 0,661 & 0,171 & 3,740 & 3,396 & 0,347 \\
\hline $\begin{array}{l}\text { Apparati elettrici e di } \\
\text { precisione }\end{array}$ & $-0,200$ & $-0,505$ & 0,318 & 0,948 & 0,729 & 0,220 & 4,337 & 3,459 & 0,870 \\
\hline Mezzi di trasporto & $-1,206$ & $-1,830$ & 0,742 & - 0,237 & - 0,850 & 0,655 & 3,209 & 2,414 & 0,789 \\
\hline $\begin{array}{l}\text { Altre industrie } \\
\text { manifatturiere }\end{array}$ & $-0,826$ & $-1,047$ & 0,236 & 0,443 & 0,188 & 0,275 & 2,005 & 1,593 & 0,416 \\
\hline
\end{tabular}

Fonte: nostre elaborazioni su dati SdC19962006.

\subsection{Le evidenze sugli indicatori di produttività: una discussione}

I risultati sin qui presentati aggiungono importanti informazioni sulla natura delle trasformazioni del sistema produttivo italiano negli anni considerati, ma al tempo stesso aprono nuove domande e problemi. Per cercare di dare una lettura alle misure di produttività ottenute, è opportuno tenere presente i cambiamenti più importanti avvenuti sui mercati del periodo in esame. Dal punto di vista dei mercati di sbocco, l'evento centrale è dato dall'ingresso dell'euro che, in un contesto di crescente globalizzazione e di ingresso di nuovi concorrenti, annulla gli spazi per l'impiego di politiche di cambio a sostegno della competitività delle merci; dal punto di vista del mercato dei fattori, l'aspetto maggiormente rilevante sembra essere la sequenza di interventi a favore dell'impiego flessibile del lavoro. Le evidenze riportate mostrano con chiarezza che l'adattamento del sistema industriale italiano alle nuove condizioni dei mercati è sensibilmente diverso nel primo periodo rispetto ai due successivi.

Quanto al primo periodo, l'aspetto sorprendente è l'arretramento della frontiera. Questo sta a significare che le imprese più efficienti, quelle che determinano la frontiera, soffrono maggiormente come conseguenza delle mutate condizioni competitive. Lo spostamento della frontiera può avvenire se le imprese individuano nuove combinazioni produttive: il fatto che non solo non 
si sperimentino nuove combinazioni, ma addirittura che le soluzioni produttive migliori sperimentate vengano sostituite da soluzioni comparativamente meno efficienti, costituisce un'evidenza problematica sotto il profilo interpretativo. Potrebbe essere accaduto che le imprese che determinano la frontiera abbiano sovrainvestito quando la domanda era trainata dalla svalutazione competitiva all'inizio degli anni 1990; oppure che le prime riforme del mercato del lavoro abbiano indotto una ristrutturazione nella composizione dei fattori che, al momento terminale del periodo in esame, era ancora in corso: in questo caso, quello che l'analisi mette in luce sarebbe la risultante di un riposizionamento in corso sulla frontiera di produzione per adattarsi al mutamento dei prezzi relativi. La scomposizione dell'indice di cambiamento tecnologico tra effetto magnitudine $(M A T E C H)$ ed effetto di composizione degli input (IBTECH) indica con chiarezza, però, che l'arretramento della frontiera dipende in misura preponderante nella generalità dei settori da un abbassamento dell'output ottenuto a fattori produttivi proporzionalmente costanti. L'impressione finale è, dunque, che le imprese migliori si siano trovate in una condizione di eccesso di capacità e siano state spiazzate dalle nuove condizioni competitive nei mercati di sbocco. In questo processo di adattamento, le imprese poste sulla frontiera potrebbero essersi trovate temporaneamente con soluzioni produttive peggiori di quelle iniziali e con ridondanze generate dai piani di costituzione di capacità produttiva già in corso d'implementazione. Il contemporaneo miglioramento dell'efficienza produttiva, invece, può essere in parte un risultato indotto dallo stesso arretramento della frontiera: se la frontiera si ritira, la posizione relativa delle imprese collocate al di sotto di essa automaticamente migliora. Ma le evidenze presentate sul ruolo dei fattori di scala nella spiegazione della componente di efficienza, dicono che questa spiegazione non è sufficiente, e che invece la riduzione dell'inefficienza sia reale e basata su un miglioramento della scala produttiva. Complessivamente si ha una duplice indicazione: da un lato sembra che il nuovo contesto competitivo blocchi i piani di espansione delle imprese più efficienti, costringendole a rivedere la propria posizione produttiva, dall'altro che induca le imprese (non necessariamente quelle poste sulla frontiera) a recuperare efficienza agendo sulla dimensione produttiva.

Nei periodi successivi, come si è visto, la storia è diversa. Probabilmente, una volta riassorbita la capacità produttiva, le imprese poste sulla frontiera riavviano un processo di innovazione che porta a uno spostamento della stessa; viceversa, le imprese rimaste indietro non tengono il passo dell'avanzamento tecnologico e perdono terreno rispetto agli innovatori allontanandosi dalla frontiera efficiente. Quest'ultimo fenomeno apre nuove questioni. Ci si può infatti chiedere se l'aumento della dispersione dell'inefficienza sia dovuto a un fenomeno di viscosità all'interno di un processo di ristrutturazione del si- 
stema produttivo che prelude all'uscita dal mercato delle imprese maggiormente inefficienti, mentre il sistema nel suo complesso segue il percorso di crescita produttiva dettato dall'avanzamento della frontiera tecnologica; ${ }^{11}$ o se in alternativa si tratti di un effetto non temporaneo che le semplici misure di distanza non riescono a catturare. Questa seconda possibilità, ricondurrebbe le dinamiche osservate all'effetto di qualche fattore non osservato.

L'ipotesi che riteniamo più credibile è che quanto emerso sia il segnale di un fenomeno non temporaneo e che i fattori non osservati siano legati a un differente comportamento strategico delle imprese. In particolare, le riforme del mercato del lavoro potrebbero avere reso accessibile lavoro flessibile e a prezzi inferiori e una parte del sistema delle imprese potrebbe avere ricercato una forma di adattamento alle nuove condizioni economiche centrata sull'impiego di lavoro della fascia meno protetta del mercato. Le evidenze emerse ci sembrano, infatti, coerenti con diversi percorsi strategici: da un lato un gruppo di imprese dinamiche, dopo avere assorbito l'effetto dell'aumento della concorrenza nei mercati finali e avere aggiustato le capacità produttive, prende una strada di innovazione tecnologica che induce uno spostamento della frontiera produttiva; dall'altra un ampio gruppo di imprese, approfittando della flessibilità introdotta nel mercato del lavoro, cerca un adattamento sul costo del lavoro, fa maggiore uso della fascia meno protetta dei lavoratori, investendo poco nel capitale umano.

L'inefficienza osservata, in questo caso, sarebbe semplicemente il risultato di una diversa scelta nella composizione del lavoro che permette alle imprese di sopravvivere agendo sul lato dei costi, ma non è catturata dalle variabili utilizzate per posizionare le imprese nell'insieme dalle possibilità produttive in cui il lavoro è considerato uniforme. All'interno degli strumenti di analisi impiegati finora, l'interpretazione delle misure ottenute risulta dunque parziale. Per questo, nella seconda parte del lavoro, si è proceduto a un secondo stadio di analisi, teso a individuare i fattori che potrebbero avere influenzato le dinamiche osservate.

\section{Dinamiche di produttività e strategie di impresa}

L'ipotesi che si intende esplorare in questa seconda parte dell'analisi è che di fronte all'evoluzione delle condizioni competitive e a un emergente dualismo del mercato del lavoro, una parte delle imprese potrebbe avere ri-

11. Su questo processo, poi, la crisi del 2008-2009 potrebbe avere agito in modo differenziato, non necessariamente penalizzando le imprese meno efficienti, come è stato dimostrato da Bugamelli, Schivardi e Zizza (2010). 
cercato una strategia di adattamento basata sull'impiego di lavoro flessibile di qualità minore e a minor costo, differentemente da altre imprese che seguono strategie centrate su investimenti in capitale umano, innovazione e crescita tecnologica.

A determinare le misure di efficienza e produttività ottenute possono concorrere sia condizioni esterne alle imprese, come l'accesso a fattori produttivi esterni, sia caratteristiche non osservate specifiche delle imprese. Un secondo stadio d'analisi di carattere econometrico in cui introdurre una qualche proxy della composizione e qualità del lavoro può permettere l'investigazione dell'ipotesi di diversi comportamenti strategici delle imprese relativamente alle scelte produttive.

Come condurre l'analisi econometrica nel secondo stadio in ambito nonparametrico è oggetto di dibattito in letteratura. Simar e Wilson (2007) pongono in luce una serie di problemi nell'impiego dell'analisi di regressione sui valori stimati di efficienza, che possono essere solo parzialmente superati ricorrendo a successive procedure di bootstrapping. Banker e Natarajan (2008) mostrano, d'altro canto, che una regressione OLS (ordinary least squares) nel secondo stadio può comunque fornire stime consistenti. In altri lavori, invece, le misure ottenute nel primo stadio sono interpretate come efficienze relative e trattate con metodi standard nell'analisi di regressione (Hoff, 2007; McDonald, 2009; Ramalho, Ramalho e Henriques, 2010). Sono state proposte infine anche analisi su variabili dipendenti qualitative costruite dalle misure di efficienza ottenute nel primo stadio (Amico Roxas, Piroli e Sorrentino, 2011; Murova e Chidmi, 2013; Thursby e Kemp, 2002).

Nel secondo stadio del nostro lavoro analizzeremo categorie di performance costruite dalle misure ottenute nel primo stadio e significative dal punto di vista interpretativo, che potrebbero risultare da differenti approcci strategici delle imprese.

L'individuazione di categorie di performance è funzionale al fatto che ciò che interessa è non semplicemente l'analisi dell'efficienza (misure di distanza) o della produttività: come abbiamo visto, in un contesto dinamico, le imprese possono modificare la propria produttività sia perché spostano la frontiera produttiva, sia perché si muovono al di sotto della stessa modificando la loro efficienza. L'uso di variabili di performance di tipo categoriale costruite su due anziché su un'unica dimensione arricchisce, a nostro giudizio, la caratterizzazione in termini strategici del comportamento delle imprese. Le imprese sono pertanto classificate lungo una dimensione statica, il livello di efficienza a inizio periodo, e una dinamica relativa al tasso di crescita della produttività. Considerando la media di ognuna delle due variabili come valore di soglia tra le categorie, ne risulta la classificazione presentata nella tab. 9. 
Tab. 9 - Numero di imprese per livello di efficienza iniziale, crescita della produttività e periodo

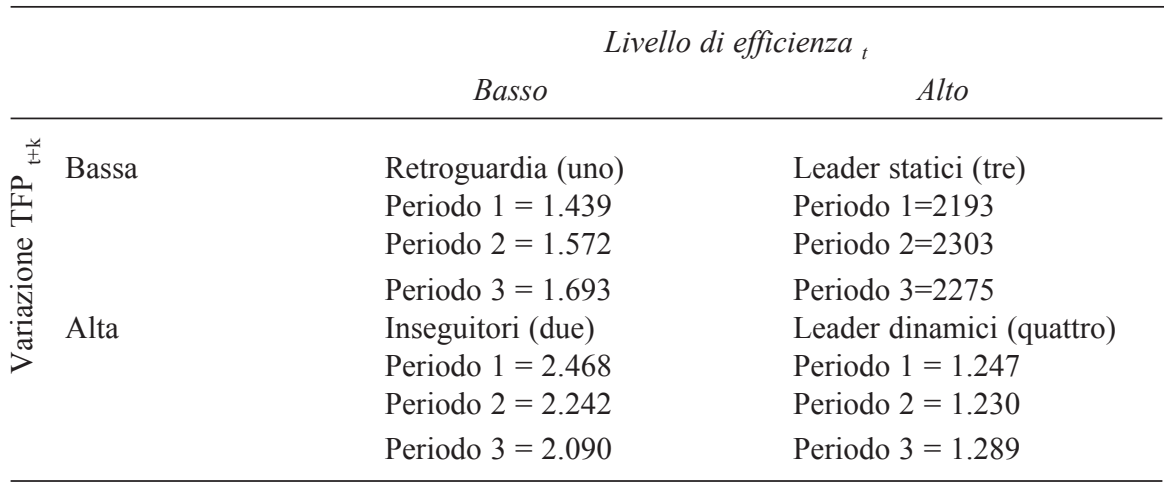

Fonte: nostre elaborazioni su dati SdC19962006.

Individuiamo così quattro categorie di imprese. La retroguardia (categoria uno) sono imprese con bassa efficienza iniziale e bassa crescita della produttività che nondimeno continuano a essere osservate nel mercato nel corso di tutto il periodo.

Gli inseguitori (categoria due) sono imprese con bassa efficienza iniziale, ma che si muovono rapidamente verso la frontiera e talvolta ne inducono anche uno spostamento: la crescita di produttività per queste imprese potrebbe essere particolarmente rapida in quanto possono agire su due leve: da un lato, il recupero di efficienza collegato a processi imitativi di imprese poste sulla frontiera e dall'altro l'avanzamento tecnologico indipendente. I leader statici (categoria tre) sono imprese vicine alla frontiera, ma con bassa dinamica della produttività e che quindi tendono, nel tempo, ad allontanarsi da questa, avendo ridotto il proprio sforzo innovativo. Infine, i leader dinamici (categoria quattro) sono le imprese poste inizialmente vicino alla frontiera tecnologica, che attraverso strategie innovative determinano uno spostamento della stessa: la crescita della produttività, in quel caso, non può avvalersi di recuperi di efficienza.

Per capire come l'appartenenza a ognuna delle categorie individuate sia influenzata da scelte strategiche relative alla composizione e qualità della forza lavoro impiegata abbiamo quindi stimato un modello logit multinomiale del tipo:

$$
P(y=j \mid \mathbf{x})=\exp \left(\mathbf{x} \beta_{j}\right) /\left[1+\sum_{k=2}^{4} \exp \left(\mathbf{x} \beta_{k}\right)\right]
$$

in cui: $j=2,3,4$ sono le categorie considerate e $\mathbf{x}$ è il vettore delle variabili esplicative e di controllo. La categoria uno (imprese di retroguardia) è quella di riferimento per la quale: 


$$
P(y=1 \mid \mathbf{x})=1 /\left[1+\sum_{k=2}^{4} \exp \left(\mathbf{x} \beta_{k}\right)\right]
$$

L'ipotesi relativa all'esistenza di diverse strategie di competizione legate al costo del lavoro e alla composizione della forza lavoro è indagata utilizzando come variabili esplicative una serie di proxy della qualità delle risorse umane impiegate dalle imprese: il costo del lavoro unitario (costo personale) se si assume che lavoro più qualificato abbia un costo in media più alto; il rapporto tra impiegati e operai (skill ratio) che ci fornisce indicazioni sulla quota di lavoratori qualificati presenti nell'impresa, nonché sul ruolo che le attività a monte e a valle del ciclo produttivo hanno nelle strategie di impresa (Bugamelli, Schivardi e Zizza, 2010); e la quota di occupati part time sul totale degli occupati (part time), una misura dell'uso di lavoro flessibile (Arvanitis, 2005), che ha un impatto sulla qualità del lavoro sotto l'assunzione che il contributo degli occupati full time sia di qualità più alta rispetto a quello degli occupati part time per motivi legati a motivazione individuale, struttura degli incentivi, livello e velocità di apprendimento sul posto di lavoro e, infine, di minore turnover tra un posto di lavoro e un altro indipendentemente dal fatto che lo spostamento sia effettuato all'interno della stessa impresa o da un'impresa a un'altra (Dolado e Stucchi, 2008). Le variabili di controllo inserite nel modello sono state scelte facendo riferimento alla letteratura che studia le determinanti della produttività d'impresa. Nel dettaglio abbiamo considerato:

1. La dimensione in termini di numero di occupati (addetti). Al riguardo Dhawan (2001), in uno studio sulle imprese americane mostra come le piccole imprese siano significativamente più produttive delle imprese più grandi suggerendo un legame negativo tra crescita della produttività e dimensione d'impresa. Recentemente, invece, Harris e Moffat (2011) mostrano come le imprese manifatturiere nel Regno Unito operino in regime di rendimenti crescenti di scala e che la dimensione d'impresa è positivamente correlata alla dinamica della produttività totale dei fattori.

2. L'età (age) delle imprese, che può avere un effetto negativo sulla dinamica della produttività se prevale l'effetto relativo alla obsolescenza della tecnologia posseduta e sfruttata dalle imprese, o positivo, se prevale, invece, l'effetto di learning-by-doing (Argote, McEvily e Reagans, 2003; Cohen e Levinthal, 1990; Harris e Moffat, 2011; Huergoa e Jaumandreu, 2004).

3. I vincoli finanziari delle imprese misurati con il cash flow (cash flow). ${ }^{12}$ La letteratura, infatti, mostra come vincoli finanziari più stringenti abbiano un effetto negativo sulle performance delle imprese in termini di crescita e profittabilità (Fagiolo e Luzzi, 2006) e sulla produttività (Bottazzi, Secchi e Tamagni, 2008; Bottazzi et al., 2011).

12. Il cash flow è calcolato come somma di: utile netto d'esercizio, ammortamenti, accantonamenti rischi e altri accantonamenti. 
4. Tre insiemi di variabili dummy per tenere conto, rispettivamente, dei periodi di analisi, dei settori di attività delle imprese e delle ripartizioni geografiche in cui le imprese operano. Tali variabili controllano per le differenti condizioni esterne in cui le imprese si trovano a operare.

Tab. 10 - Statistiche descrittive

\begin{tabular}{|c|c|c|c|c|c|c|c|c|}
\hline \multirow[t]{2}{*}{ Variabile } & \multicolumn{2}{|c|}{ Retroguardia } & \multicolumn{2}{|c|}{ Leader statici } & \multicolumn{2}{|c|}{ Inseguitori } & \multicolumn{2}{|c|}{ Leader dinamici } \\
\hline & Media & Dev. std. & Media & Dev. std. & Media & Dev. std. & Media & Dev. std. \\
\hline $\begin{array}{l}\text { Costo_personale } \\
(\mathrm{mgl} . €)\end{array}$ & 20,700 & 5,200 & 23,800 & 7,400 & 20,000 & 5,600 & 23,800 & 7,500 \\
\hline $\begin{array}{l}\text { Skill ratio } \\
\text { (rapporto) }\end{array}$ & 0,460 & 1,420 & 0,660 & 2,530 & 0,470 & 1,280 & 0,880 & 3,510 \\
\hline $\begin{array}{l}\text { Part time } \\
\text { (rapporto) }\end{array}$ & 0,043 & 0,060 & 0,037 & 0,053 & 0,041 & 0,058 & 0,039 & 0,056 \\
\hline $\begin{array}{l}\text { Cash flow } \\
\text { (mgl. } € \text { ) }\end{array}$ & 393,000 & 723,000 & 649,800 & $1.246,600$ & 327,700 & 576,600 & 564,300 & $1.151,800$ \\
\hline $\begin{array}{l}\text { Addetti } \\
\text { (unità) }\end{array}$ & 54,500 & 52,900 & 47,500 & 52,800 & 53,000 & 49,600 & 44,000 & 46,600 \\
\hline $\begin{array}{l}\text { Età } \\
\text { (anni) }\end{array}$ & 22,500 & 12,400 & 21,700 & 13,200 & 21,100 & 12,400 & 20,900 & 12,700 \\
\hline
\end{tabular}

Fonte: nostre elaborazioni su dati SdC19962006.

Tab. 11 - Matrice delle correlazioni

Variabile Costo_personale Skill ratio Part time Cashflow Addetti Età

\begin{tabular}{lrlllll}
\hline Costo_personale & \multicolumn{1}{c}{1} & & & & & \\
Skill ratio & $0,167^{*}$ & 1 & & & & \\
Part time & $-0,141^{*}$ & $0,034^{*}$ & 1 & & & \\
Cash flow & $0,225^{*}$ & $0,078^{*}$ & $-0,075^{*}$ & 1 & & \\
Addetti & $0,147^{*}$ & $-0,009$ & $-0,065^{*}$ & $0,597^{*}$ & 1 & \\
Età & $0,256^{*}$ & 0,007 & $0,038^{*}$ & $0,092^{*}$ & 0,158 & 1 \\
\hline
\end{tabular}

${ }^{*}$ significatività $5 \%$.

Fonte: nostre elaborazioni su dati SdC19962006.

Nelle tab. 10 e 11 sono riportate media e deviazione standard delle variabili esplicative e la matrice delle correlazioni. Il gruppo di retroguardia ha in media un più alto numero di addetti (cinquantaquattro virgola cinque), utilizza più part time (zero virgola zero quattro) e ha un'età (ventidue virgola cinque) più elevata rispetto alle imprese delle altre categorie. Il gruppo dei leader dinamici, sperimenta invece un costo del personale più alto (ventitre virgola otto), ha un cash flow più elevato (seicentocinquanta) e utilizza forza lavoro con più alta intensità di skill (zero virgola ottantotto) e ha un'età più bassa rispetto a ogni altro gruppo. 
Si noti infine come i leader statici assomiglino a quelli dinamici quanto a costo del personale e bassa presenza di part time. Hanno tuttavia un cash flow superiore e uno skill ratio inferiore. La matrice delle correlazioni mostra come il numero di addetti e il cash flow siano positivamente correlati (zero virgola sei), mentre per tutte le altre coppie di variabili esplicative la correlazione appare molto bassa. La tab. 12 presenta il modello stimato usando diversi insiemi di variabili esplicative. In tutte le specificazioni proposte è inoltre considerato l'intero set di controlli sui vincoli finanziari, sulla dimensione ed età dell'impresa, e le dummy periodo, settore e localizzazione geografica. Le stime riportate si riferiscono ai log-odds ratio, ovvero il logaritmo della variazione della probabilità di classificazione nella categoria $(j=2,3,4)$ rispetto alla categoria di riferimento (uno) attesa per effetto della variazione unitaria della variabile esplicativa considerata. Il modello 1 usa solo il costo unitario del lavoro (costo personale). Un diverso costo del lavoro, però, può rappresentare differenti qualità del fattore produttivo, oppure, in presenza di un mercato del lavoro segmentato, può risultare dall'impiego di lavoro della stessa qualità ad un prezzo inferiore. Questa seconda ipotesi è consistente con l'idea di mercato del lavoro a due livelli presentata da Boeri e Garibaldi (2007).

L'effetto atteso sulla misura di inefficienza produttiva è tuttavia diverso nei due casi. Infatti, mentre l'omissione di un fattore non osservabile (differente qualità del lavoro impiegato) ha un effetto sulla misura di inefficienza delle imprese che usano lavoro di qualità inferiore, il semplice differenziale di costo dovrebbe comportare un aggiustamento della composizione dei fattori che non ha implicazioni sull'efficienza tecnica, bensì solo sull'efficienza allocativa. L'evidenza di una relazione tra costo del lavoro ed efficienza dovrebbe dunque supportare l'ipotesi che le riforme del mercato del lavoro abbiano determinato una differenziazione non solo di prezzo, ma anche nella scelta della composizione qualitativa del lavoro. Per rafforzare l'ipotesi che non si tratti di semplice adattamento al costo dei fattori, ma di differenti scelte della composizione della forza lavoro nelle imprese, nel modello 2 sono introdotti il rapporto impiegati/operai (skill ratio) e il rapporto tra addetti part time e addetti totali (part time). Il modello 3, infine, introduce un'interazione tra costo del lavoro e i diversi intervalli temporali: periodo due dall'anno 2000 al 2003 e periodo tre dall'anno 2003 al 2006.

Dalla tab. 12 possiamo osservare che, in tutte le specificazioni adottate, il costo del lavoro ha una relazione positiva con la probabilità di appartenere ai gruppi dei leader, statici o dinamici, rispetto al gruppo base delle imprese di retroguardia (categoria uno). Lo skill ratio è particolarmente significativo nel contraddistinguere l'appartenenza al gruppo delle imprese dinamiche, mentre la presenza di addetti part time riduce la probabilità di appartenere a ognuno dei gruppi diversi dalle imprese di retroguardia. 
E. Tundis, E. Zaninotto, R. Gabriele, S. Trento Produttività delle imprese italiane: 1996-2006

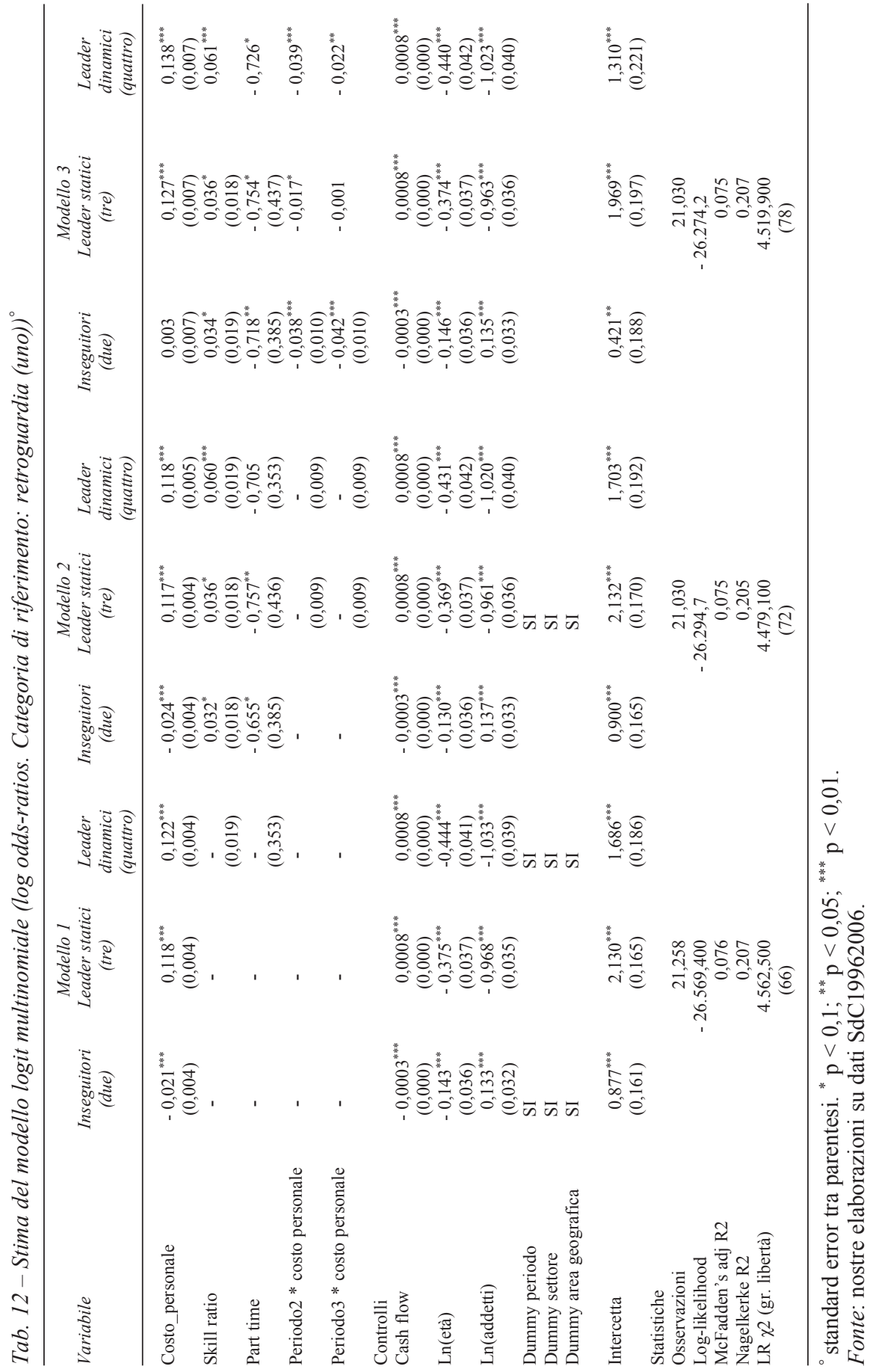


Per quanto riguarda le variabili di controllo, osserviamo che rispetto alla categoria di riferimento (retroguardia):

1. il cash flow aumenta la probabilità di classificazione nella categoria dei leader (statici e dinamici) e riduce la probabilità di appartenenza alla terza degli inseguitori, ma con un effetto che, pur essendo significativo, è molto contenuto;

2. una maggiore età dell'impresa diminuisce la probabilità di appartenere al gruppo dei leader;

3. un aumento della dimensione agisce negativamente sulla probabilità di appartenere alle categorie delle imprese poste vicino alla frontiera.

La dimensione appare quindi negativamente correlata all'efficienza, ovvero alla distanza dalla frontiera. L'effetto negativo della dimensione di impresa sembra andare in senso contrario a una parte consistente della letteratura che viceversa evidenzia un ruolo positivo della dimensione sulla produttività. È tuttavia possibile, come già abbiamo intuito dall'analisi delle componenti della produttività che nei periodi presi in esame si sia manifestato un processo di ridimensionamento dei piani di sviluppo delle imprese efficienti. ${ }^{13}$

Per dare un'idea più precisa dell'impatto delle variabili sulla probabilità di appartenere a una determinata classe, la tab. 13 presenta, per il solo modello 3, l'effetto marginale di una variabile sulla probabilità di appartenere a ogni singola categoria, misurata come di consueto attorno alla media delle variabili.

Per quanto riguarda la consistenza dei risultati con la nostra ipotesi di lavoro osserviamo che all'aumentare del costo unitario del lavoro (costo personale) aumenta la probabilità di appartenenza al gruppo delle imprese "di frontiera" (categorie tre e quattro) e diminuisce quella di appartenere alla retroguardia e agli inseguitori. L'effetto è significativo in termini di variazione. L'effetto marginale della variazione del costo del lavoro, sebbene permanga negativo in tutti i periodi, mostra una diversa evoluzione nel tempo relativamente all'effetto sulla probabilità di appartenenza alle imprese di retroguardia (categoria uno) e agli inseguitori (categoria due): nel primo caso si osserva una tendenziale riduzione dell'impatto negativo mentre si ha un'amplificazione nel secondo. Ciò sembra suggerire che, dopo la fase di adattamento all'euro (quella contrassegnata da retrocessione tecnologica e aumento di efficienza), le imprese inseguitrici sembrano effettivamente percorrere un modello di riduzione del costo del lavoro unitario rispetto alle imprese della frontiera.

13. Questi risultati sull'effetto dell'età e della dimensione di impresa sulla dinamica produttiva sono peraltro coerenti con quelli individuati da Hall, Lotti e Mairesse (2009) sull'effetto dell'innovazione sulla produttività tra le piccole e medie imprese italiane e di Dhawan (2001) sugli Stati Uniti. Una relazione negativa tra dimensione ed efficienza produttiva emerge anche in Diaz e Sanchez (2008) nel caso di imprese spagnole. 
Tab. 13 - Stima degli effetti marginali (effetto della variazione unitaria dei regressori sulla probabilità di appartenere alla categoria j-esima ${ }^{\circ}$

\begin{tabular}{|c|c|c|c|c|}
\hline \multirow[b]{2}{*}{ Variabile } & \multicolumn{4}{|c|}{ Modello 3} \\
\hline & $\begin{array}{l}\text { Retroguardia } \\
\text { (uno) }\end{array}$ & $\begin{array}{c}\text { Inseguitori } \\
\text { (due) }\end{array}$ & $\begin{array}{c}\text { Leader statici } \\
\text { (tre) }\end{array}$ & $\begin{array}{c}\text { Leader } \\
\text { dinamici } \\
\text { (quattro) }\end{array}$ \\
\hline Costo_personale & $\begin{array}{c}-0,0141^{* * *} \\
(0,0010)\end{array}$ & $\begin{array}{c}-0,0181^{* * *} \\
(0,0011)\end{array}$ & $\begin{array}{l}0,0195^{* * *} \\
(0,0010)\end{array}$ & $\begin{array}{l}0,0128^{* * *} \\
(0,0007)\end{array}$ \\
\hline Skill ratio & $\begin{array}{c}-0,0070^{* *} \\
(0,0030)\end{array}$ & $\begin{array}{c}0,0006 \\
(0,0024)\end{array}$ & $\begin{array}{c}0,0014 \\
(0,0021)\end{array}$ & $\begin{array}{l}0,0050^{* * *} \\
(0,0012)\end{array}$ \\
\hline Part time & $\begin{array}{l}0,1260^{* *} \\
(0,0542)\end{array}$ & $\begin{array}{l}-0,0432 \\
(0,0617)\end{array}$ & $\begin{array}{r}-0,0566 \\
(0,0659)\end{array}$ & $\begin{array}{r}-0,0265 \\
(0,0515)\end{array}$ \\
\hline Periodo $2 *$ costo personale & $\begin{array}{l}0,0051^{* * *} \\
(0,0013)\end{array}$ & $\begin{array}{c}-0,0045^{* * *} \\
(0,0014)\end{array}$ & $\begin{array}{c}0,0020 \\
(0,0013)\end{array}$ & $\begin{array}{c}-0,0027^{* * *} \\
(0,0010)\end{array}$ \\
\hline Periodo $3 *$ costo personale & $\begin{array}{l}0,0043^{* * *} \\
(0,0013)\end{array}$ & $\begin{array}{c}-0,0068^{* * *} \\
(0,0015)\end{array}$ & $\begin{array}{l}0,0030^{* *} \\
(0,0014)\end{array}$ & $\begin{array}{l}-0,0005 \\
(0,0010)\end{array}$ \\
\hline Controlli & & & & \\
\hline Cash flow & $\begin{array}{c}-7,26 \mathrm{e}-05^{* * *} \\
(6,38 \mathrm{e}-06)\end{array}$ & $\begin{array}{c}-0,0002^{* * *} \\
(8,02 \mathrm{e}-06)\end{array}$ & $\begin{array}{c}0,0002^{* * *} \\
(6,18 \mathrm{e}-06)\end{array}$ & $\begin{array}{l}8,79 \mathrm{e}-05^{* * *} \\
(4,06 \mathrm{e}-06)\end{array}$ \\
\hline Ln(età) & $\begin{array}{l}0,0520^{* * * *} \\
(0,0055)\end{array}$ & $\begin{array}{l}0,0264^{* * *} \\
(0,0058)\end{array}$ & $\begin{array}{c}-0,0431^{* * *} \\
(0,0059)\end{array}$ & $\begin{array}{c}-0,0352^{* * *} \\
(0,0046)\end{array}$ \\
\hline Ln(addetti) & $\begin{array}{l}0,0961^{* * *} \\
(0,0049)\end{array}$ & $\begin{array}{c}0,169^{* * *} \\
(0,0056)\end{array}$ & $\begin{array}{c}-0,164^{* * *} \\
(0,0057)\end{array}$ & $\begin{array}{c}-0,101^{* * *} \\
(0,0043)\end{array}$ \\
\hline
\end{tabular}

${ }^{\circ}$ controlli periodo, settore e area geografica sono considerati. Standard error tra parentesi. ${ }^{*} \mathrm{p}<0,1 ;{ }^{* *} \mathrm{p}<0,05 ;{ }^{* * *} \mathrm{p}<0,01$.

Fonte: nostre elaborazioni su dati SdC19962006.

Il rapporto impiegati/operai (skill ratio) sembra agire nella direzione attesa, ed è significativo nello spiegare la probabilità di appartenenza al gruppo dei leader dinamici. In termini di variazioni, questa variabile sembra avere effetto soprattutto sulle categorie estreme, con un impatto significativo di aumento della probabilità di rientrare tra $\mathrm{i}$ leader dinamici e di ridurre quella di rientrare nella categoria delle imprese di retroguardia. Questo aspetto ci sembra particolarmente significativo alla luce del ruolo che l'investimento in capitale umano nelle fasi a monte (progettazione e disegno dei prodotti) e a valle (marketing e commercializzazione) sembra avere sulla competitività delle imprese italiane (Bugamelli, Schivardi e Zizza, 2010). La quota dei part time sul totale degli addetti (part time) aumenta la probabilità di appartenenza al gruppo di retroguardia che sembra attingere in misura maggiore delle altre imprese alla fascia flessibile del mercato del lavoro, approssimata dal ricorso al part time.

Nel complesso dunque si può ritenere che le imprese della retroguardia, 
siano caratterizzate mediamente da un'età maggiore e adottino una strategia difensiva, impiegando lavoro di minore qualità e ricorrendo al segmento non protetto del mercato del lavoro. Le imprese inseguitrici sembrano usare una mistura di strategie basate sull'estensione dell'attività e sul lavoro meno qualificato per rincorrere posizioni vicine alla frontiera, ma con una combinazione di fattori favorevole al lavoro. Le imprese efficienti, indipendentemente dalla dinamica di produttività, usano lavoro più qualificato, ma quelle più dinamiche sono anche maggiormente terziarizzate.

L'esercizio svolto, ovviamente, può dare solo alcune indicazioni sulla natura strategica dell'eterogeneità che si osserva nelle performance produttive. Il problema dell'endogeneità di alcuni controlli potrebbe rappresentare un limite alla validità dei risultati, ma si osservi che le variabili esplicative utilizzate fanno riferimento ai livelli iniziali di ciascun periodo proprio per mitigare questo problema. Le regolarità osservate, fanno pensare comunque che la natura strategica dell'eterogeneità osservata abbia un fondamento e che diversi gruppi di imprese stiano percorrendo modelli di adattamento diversi alle nuove condizioni di mercato.

\section{Conclusione}

Le prime analisi sulle persistenti difficoltà di crescita dell'economia italiana negli ultimi venti anni sembravano sottolineare l'esistenza di una generalizzata incapacità del sistema produttivo a raccogliere le sfide poste dalla crescente globalizzazione dei mercati e dalla maggiore disciplina imposta dalla moneta unica europea.

L'analisi condotta in questo lavoro sembra invece evidenziare come dietro alla bassa crescita della produttività che ha caratterizzato l'Italia a partire all'incirca dal 1995 si celi una elevata eterogeneità delle imprese. L'industria manifatturiera subisce un profondo shock nella seconda metà del decennio 1990 ed è ragionevole pensare, coerentemente con diversi studi citati, che lo shock sia in larga misura da ricondurre al cambiamento del gioco competitivo conseguente all'ingresso dell'euro e all'impossibilità di sfruttare la leva della svalutazione competitiva in un momento in cui, contemporaneamente, si accentua la concorrenza internazionale.

Successivamente però, e soprattutto dopo la fase negativa del 2000-2003, si hanno evidenti segni di un recupero dell'industria manifatturiera italiana. ${ }^{14}$ I riscontri che abbiamo presentato al riguardo sembrano coerenti con quelli raggiunti da altri lavori, svolti con diverse metodologie. L'approccio da noi segui-

14. Sull'effetto della crisi sull'innovazione delle imprese, si veda il recente lavoro di Pianta e Lucchese (2011). 
to permette di isolare con maggiore precisione la componente della crescita della produttività dovuta al cambiamento tecnologico delle imprese esistenti. $\mathrm{Ne}$ emerge l'evidenza di un dualismo marcato presente nel nostro sistema produttivo. Da un lato si osserva una ripresa della dinamica di crescita produttiva, ma al tempo stesso vi sono prove di una difficoltà delle imprese a mantenere il passo con il gruppo di innovatori, con una conseguente perdita in termini di efficienza rispetto alla frontiera tecnologica.

Nell'ultima parte del lavoro ci siamo poi chiesti se tale dinamica costituisca un fenomeno transitorio entro un percorso di aggiustamento del mercato, o possa invece essere collegata a diversi modelli strategici di adattamento. Le evidenze che abbiamo segnalato rafforzano l'ipotesi che le imprese stiano percorrendo diversi sentieri di adattamento e che un ruolo decisivo dei diversi percorsi sia dato dall'impiego del lavoro. Le riforme del mercato del lavoro realizzate in Italia negli anni novanta hanno infatti ridotto in modo drastico il costo d'uso e forse la qualità di una fascia di lavoratori di nuova assunzione. La disponibilità di manodopera "flessibile" meno costosa ma anche meno abile ha rappresentato per alcune categorie d'imprese una facile soluzione alle difficoltà competitive. Al contrario, le imprese maggiormente efficienti e dinamiche (i leader dinamici) sono al tempo stesso imprese che hanno un costo unitario del lavoro più elevato, sono maggiormente terziarizzate, si avvalgono meno del segmento "flessibile" del mercato del lavoro, e sono anche imprese più giovani e di dimensioni minori. All'estremo opposto stanno le imprese di retroguardia. L'eterogeneità dei comportamenti potrebbe, in questo quadro, essere un po' più leggibile, segnalando diversi pattern adattivi presenti nella manifattura italiana. Rispetto ad altri lavori che evidenziano la relazione tra le riforme del mercato del lavoro e la caduta di produttività industriale, la nostra ricerca mette in luce come questa relazione non sia uniforme, bensì ingeneri una sorta di neo-dualismo nella manifattura italiana.

La presenza di un gruppo consistente di imprese dinamiche è certamente una evidenza rassicurante per il futuro del nostro sistema industriale. D'altra parte, però, le riforme imperfette del mercato del lavoro hanno offerto alle imprese meno dinamiche ampi spazi di adattamento che permettono loro di rimanere nel mercato a dispetto di una crescente distanza dalla frontiera tecnologica. Il problema per l'economia italiana sembra dunque essere quello di diffondere le migliori pratiche produttive a tutto il sistema, evitando la trappola di un dualismo persistente che deprime la crescita. È difficile nondimeno dire quale spazio di persistenza possano avere i modelli di adattamento basati sull'impiego di lavoro meno qualificato e costoso. La natura dell'analisi effettuata, basata su un panel di imprese lungo undici anni, non può che osservare le imprese che sono sopravvissute e non è in grado di dire niente sulla dinamica di entrata e uscita dal mercato e di crescita connessa alle diverse strategie produttive 
delle imprese. Ė noto come la dinamica demografica delle imprese sia un fattore fondamentale nella spiegazione della crescita della produttività (Bartelsman, Hartiwanger e Scarpetta, 2009): sarebbe necessario muoversi anche in questa direzione complementare per comprendere meglio le origini della lunga stagnazione della produttività italiana.

\section{Riferimenti bibliografici}

Aiello F., Pupo V., Ricotta F. 2009. Sulla dinamica della produttività totale dei fattori in Italia. Un'analisi settoriale. L'Industria, 30 (3): 413-435.

Amico Roxas S., Piroli G., Sorrentino M. 2011. Efficiency and evaluation analysis of a network of technology transfer brokers. Technology Analysis \& Strategic Management, 23 (1): 7-24.

Argote L., McEvily B., Reagans R. 2003. Managing knowledge in organizations: an integrative framework and review of emerging themes. Management Science, 49 (4): 571-82.

Arvanitis S. 2005. Modes of labor flexibility at firm level: are there any implications for performance and innovation? Evidence for the Swiss economy. Industrial and Corporate Change, 14 (6): 993-1016.

Banker R.D., Charnes A., Cooper W.W. 1984. Some models for estimating technical and scale inefficiencies in data envelopment analysis. Management Science, 30 (9): 1078-1092.

Banker R.D., Natarajan R. 2008. Evaluating contextual variables affecting productivity using data envelopment analysis. Operations Research, 56 (1): 48-58.

Bartelsman E.J., Haltiwanger J.C., Scarpetta S. 2009. Cross-country differences in productivity: the role of allocation and selection. NBER Working Paper 15490.

Blanchard O., Landier A. 2002. The perverse effects of partial labour market reform: fixed-term contracts in France. The Economic Journal, 112 (480): F214-F244.

Boeri T., Garibaldi P. 2007. Two tier reforms of employment protection: a honeymoon effect?. The Economic Journal, 117 (521): F357-F385.

Bottazzi G., Secchi A., Tamagni F. 2008. Productivity, profitability and financial performance. Industrial and Corporate Change, 17 (4): 711-751.

Bottazzi G., Grazzi M., Secchi A., Tamagni F. 2011. Financial and economic determinants of firm default. Journal of Evolutionary Economics, 21 (3): 373-406.

Brandolini A., Cipollone P. 2001. Multifactor productivity and labour quality in Italy, 1981-2000. Temi di Discussione della Banca d'Italia 422.

Brandolini A., Bugamelli M. 2009. Rapporto sulle tendenze nel sistema produttivo italiano. Questioni di economia e di finanza della Banca d'Italia 45.

Bugamelli M. 2007. Prezzi delle esportazioni, qualità dei prodotti e caratteristiche di impresa: un'analisi su un campione di imprese italiane. Temi di discussione della Banca d'Italia 634.

Bugamelli M., Schivardi F., Zizza R. 2010. The euro and firm restructuring, in Alesina A., Giavazzi F. (eds.) Europe and the euro. University of Chicago Press: Chicago.

Charnes A., Cooper W.W., Rhodes E. 1978. Measuring the efficiency of decisionmaking units. European Journal of Operational Research, 2 (6): 429-444. 
Ciocca P. 2004. L'economia italiana: un problema di crescita. Rivista Italiana degli Economisti, 9 (1, supplemento): 7-28.

Coelli T.J., Rao D.S.P., O'Donnell C.J., Battese G.E. 2005. Introduction to Efficiency and Productivity Analysis. Springer: New York.

Cohen W.M., Levinthal D.A. 1990. Absorptive-capacity. A new perspective on learning and innovation. Administrative Science Quarterly, 35 (1): 128-152.

D'Antonio M., Scarlato M. 2004. Trent'anni di trasformazioni dell'economia italiana: verso la ripresa dello sviluppo?. Economia Italiana, 2: 277-331.

Daveri F. 2008. Torniamo a dare i numeri sulla produttività. Lavoce.info, published online.

Daveri F., Jona-Lasino C. 2005. Italy's decline: getting the facts right. Giornale degli Economisti e Annali di Economia, 64 (4): 365-410.

Dhawan R. 2001. Firm size and productivity differential: theory and evidence from a panel of U.S. firms. Journal of Economic Behavior \& Organization, 44 (3): 269-293.

Diaz M.A, Sanchez R. 2008. Firm size and productivity in Spain: a stochastic frontier analysis. Small Business Economics, 30 (3): 315-323.

Dolado J.J., Stucchi R. 2008. Do temporary contracts affect TFP? Evidence from Spanish manufacturing firms. IZA Discussion Paper 3832.

Dosi G., Grazzi M., Tomasi C., Zeli A. 2011. Turbolence underneath the big calm? Exploring the micro-evidence behind the flat trend of manufacturing productivity in Italy. Small Business Economics, published online.

Fagiolo G., Luzzi A. 2006. Do liquidity constraints matter in explaining firm size and growth? Evidence from the Italian manufacturing industry. Industrial and Corporate Change, 15 (1): 1-39.

Faini R. 2004. Fu vero declino? L'Italia negli anni '90, in Toniolo G, Visco V. (a cura di) Il declino economico dell'Italia: cause e rimedi. Bruno Mondadori: Milano.

Faini R., Sapir A. 2005. Un modello obsoleto? Crescita e specializzazione dell'economia italiana, in Boeri T., Faini R., Ichino A., Pisauro G., Scarpa C. (a cura di) Oltre il declino. il Mulino: Bologna.

Färe R., Grosskopf S., Norris M., Zhongyang Z. 1994. Productivity growth, technical progress and efficiency change in industrialised countries. The American Economic Review, 84 (1): 66-83.

Färe R., Grosskopf S. 1996. Intertemporal Production Frontiers: With Dynamic DEA. Kluwer Academic Publishers: Boston.

Foresti G., Guelpa F., Trenti S. 2007. Quali leve per il rilancio dell'industria? La questione dimensionale. Intesa Sanpaolo: Milano.

Hall B.H., Lotti F., Mairesse J. 2009. Innovation and productivity in SMEs. Empirical evidence for Italy. Small Business Economics, 33 (1): 13-33.

Harris R., Moffat J. 2011. Plant-level determinants of total factor productivity in Great Britain, 1997-2006. SERC Discussion Papers 64.

Hoff A. 2007. Second stage DEA: comparison of approaches for modelling the DEA score. European Journal of Operational Research, 181 (1): 425-435.

Huergoa E., Jaumandreu J. 2004. Firms' age, process innovation and productivity growth. International Journal of Industrial Organization, 22 (4): 541-559.

IMF 2007. Italy-Selected Issues, IMF Country Report 07/65. IMF Publication Service: Washington. 
Istat. 2001. $8^{\circ}$ Censimento industria e servizi. Istat: Roma.

Lucidi F. 2008. Labour market flexibility and productivity growth: new evidence from firm-level data. Paper presented at the XXIII National Conference of Labour Economics. Università di Brescia, September 11-12.

McDonald J. 2009. Using least squares and tobit in second stage DEA efficiency analysis. European Journal of Operational Research, 197 (2): 792-798.

Milana C., Nascia L., Zeli A. 2008. Changes in multifactor productitvity in Italy from 1998 to 2004: evidence from firm-level data using DEA. EU KLEMS Working Paper Series 33.

Murova O., Chidmi B. 2013. Technical efficiency of U.S. dairy farms and federal government programs. Applied Economics, 45 (7): 839-847.

OECD 2007. Economic Survey of Italy. OECD: Paris.

Pedrotti L., Tundis E., Zaninotto E. 2008. Crescita economica e produttività: misure e applicazioni. Il caso della provincia autonoma di Trento. Edizioni 31: Trento.

Pianta M., Lucchese M. 2011. Crisis, cycles and innovation. Paper presented at the conference Crises, Institutions and Labour Market Performance: Comparing Evidence and Policies. Università di Perugia, November 10.

Ramalho E.A., Ramalho J.J.S., Henriques P.D. 2010. Fractional regression models for second stage DEA efficiency analysis. Journal of Productivity Analysis, 34 (3): 239-255.

Sampaio de Sousa M.C., Stosic B. 2005. Technical efficiency of the Brazilian municipalities: correcting nonparametric frontier measurement for outliers. Journal of Productivity Analysis, 24 (2): 157-181.

Simar L., Wilson P.W. 2007. Estimation and inference in two-stage, semi-parametric models of production processes. Journal of Econometrics, 136 (1): 31-64.

Simar L., Wilson P.W. 2008. Statistical inference in nonparametric frontier models: recent development and perspectives, in Fried H., Lovell C.A.K., Schmidt S. (eds.) The Measurement of Productive Efficiency. Oxford University Press: London.

Thursby J.G., Kemp S. 2002. Growth and productive efficiency of university intellectual property licensing. Research Policy, 31 (1): 109-124.

Toniolo G. 2004. L'Italia verso il declino economico? Ipotesi e congetture in una prospettiva secolare. Rivista Italiana degli Economisti, 9 (1, supplemento): 29-46.

Tronti L. 2010. The Italian productivity slow-down: the role of the bargaining model. International Journal of Manpower, 31 (7): 770-792.

Wheelock D.C., Wilson P.W. 1999. Inefficiency and productivity change in U.S. banking. 1984-1993. Journal of Money, Credit and Banking, 31 (2): 212-234. 